Published in final edited form as:

Nat Methods. 2016 September ; 13(9): 763-769. doi:10.1038/nmeth.3935.

\title{
A far-red fluorescent protein evolved from a cyanobacterial phycobiliprotein
}

\author{
Erik A. Rodriguez ${ }^{1,6}$, Geraldine N. Tran ${ }^{2,6}$, Larry A. Gross ${ }^{3}$, Jessica L. Crisp ${ }^{1}$, Xiaokun \\ Shu ${ }^{4}$, John Y. Lin ${ }^{5}$, and Roger Y. Tsien ${ }^{1,3}$ \\ ${ }^{1}$ Department of Pharmacology, University of California, San Diego, La Jolla, California, USA \\ ${ }^{2}$ School of Medicine, University of California, San Francisco, San Francisco, California, USA \\ ${ }^{3}$ Howard Hughes Medical Institute, La Jolla, California, USA \\ ${ }^{4}$ Department of Pharmaceutical Chemistry and Cardiovascular Research Institute, University of \\ California, San Francisco, San Francisco, California, USA \\ ${ }^{5}$ University of Tasmania, Private Bag 23, 7000 Hobart, Tasmania, Australia
}

\begin{abstract}
Far-red fluorescent proteins (FPs) are desirable for in vivo imaging because less light is scattered, absorbed, or reemitted by endogenous biomolecules. A new class of FP was developed from an allophycocyanin a-subunit (APCa). Native APC requires a lyase to incorporate phycocyanobilin. The evolved FP, named small $U$ tra-Red FP(smURFP), covalently attaches biliverdin (BV) without a lyase, and has 642/670 nm excitation/emission peaks, a large extinction coefficient $\left(180,000 \mathrm{M}^{-1} \mathrm{~cm}^{-1}\right)$ and quantum yield (18\%), and comparable photostability to eGFP. SmURFP has significantly increased BV incorporation rate and protein stability compared to the bacteriophytochrome (BPH) FPs. BV supply is limited by membrane permeability, so expression of heme oxygenase-1 with heme precursors increases fluorescence of BPH/APCa FPs. SmURFP (but not BPH FPs) can incorporate a more membrane-permeant BV analog, making smURFP fluorescence in situ comparable to FPs from jellyfish/coral. A far-red/near-infrared fluorescent cell cycle indicator was created with smURFP and a BPH FP.
\end{abstract}

Users may view, print, copy, and download text and data-mine the content in such documents, for the purposes of academic research, subject always to the full Conditions of use:http://www.nature.com/authors/editorial_policies/license.html\#terms

Correspondence should be addressed to R.Y.T. (rtsien@ucsd.edu).

${ }^{6}$ E.A.R. and G.N.T. contributed equally to this work.

Accession codes. GenBank/EMBL/DDBJ: smURFP, KX449134; TDsmURFP, KX449135. Bacterial and mammalian expression plasmids and lentiviral transfer vectors are available at Addgene: smURFP, 80341, 80343, 80345, 80347, 80348, 80349; and TDsmURFP, 80342, 80344, 80346.

\section{AUTHOR CONTRIBUTIONS}

E.A.R and G.N.T. created bacterial expression plasmids, evolved and developed smURFP/APCaFPs in Escherichia coli, and characterized properties. E.A.R. prepared mammalian plasmids, made smURFP homology model, created TDsmURFP, performed BV incorporation rates, performed mammalian cell experiments (HO-1, different chromophores, protein stability, and FP photobleaching), created FR/NIR FUCCI, and performed fluorescence imaging in vitro \& in vivo. J.Y.L. transduced neurons. E.A.R. and J.Y.L. created virus and stable HT1080 cells for mouse models. J.Y.L. and J.L.C. injected animals with cancer cells and chromophores. J.L.C. prepared plasma. E.A.R., G.N.T., and J.Y.L. purified PCB and analyzed data. L.A.G. performed MS. X.S. chose the Trichodesmium APCa gene and oversaw the first three rounds of evolution of APCa+PCB. R.Y.T. and E.A.R. oversaw the design and analysis of the experiments. All authors contributed to writing and discussion.

COMPETING FINANCIAL INTERESTS

The authors declare no competing financial interests. 
FPs enable tracking of gene expression, cell fate, and fusion proteins ${ }^{1-4}$. FPs from jellyfish/ coral are spectrally limited to excitation maxima $<610 \mathrm{~nm}$, require $\mathrm{O}_{2}$, and produce $\mathrm{H}_{2} \mathrm{O}_{2}$ upon chromophore formation, thus requiring an aerobic environment tolerant of reactive oxygen species ${ }^{5}$. Chromophore formation may take hours ${ }^{5}$, and mixtures of green/red fluorescence are common ${ }^{6-8} \cdot \mathrm{H}_{2} \mathrm{O}_{2}$ is a mediator of cell survival, growth, differentiation, and implicated in diseases ${ }^{9-12}$, which could complicate experimental results. These problems inspire researchers to evolve FPs using endogenous chromophores to eliminate $\mathrm{O}_{2}$ necessity ${ }^{13}$ and $\mathrm{H}_{2} \mathrm{O}_{2}$ production. Far-red (FR)/near-infrared (NIR) FPs are desirable for imaging in living animals because these wavelengths minimize light scattering and absorbance by endogenous biomolecules, which reduces autofluorescence ${ }^{14}$. NIR FPs were engineered from nonfluorescent bacteriophytochromes (BPHs) that attach BV, but have low quantum yield (QY) and protein stability ${ }^{15-20}$.

We started from the light harvesting phycobiliproteins from cyanobacteria (APCa from Trichodesmium erythraeum (TeAPCa)). Native APC is a highly fluorescent hexamer (three $a+\beta$ dimers) and uses an auxiliary protein, known as a lyase, to incorporate phycocyanobilin (PCB, Fig. 1a-c) $)^{21}$. Native APC biliprotein FPs were created, but required a lyase, used PCB, and were expressed only in Escherichia coli ${ }^{22,23}$. First, TeAPCa mutants were evolved to autocatalytically attach PCB (without a lyase) and fluoresce. Second, PCB is not present in mammals, so we evolved FPs that bind BV (Fig. 1c), a molecule ubiquitous to eukaryotes and produced at $300-500 \mathrm{mg} / \mathrm{day}$ in humans ${ }^{24}$. After 12 rounds of mutating and screening $\sim 10^{6}$ bacterial colonies, a FP was selected with 20 mutations, named smURFP (homodimer lacking chromophore, Fig. 1d).

\section{RESULTS}

\section{Engineering and characterization of APCaFPs}

We chose TeAPCa (15 kD) because it lacked 29 amino acids common to other APCa (Supplementary Fig. 1). Expression of TeAPCa with heme oxygenase-1 (HO-1) and phycocyanobilin:ferredoxin oxidoreductase (PcyA), for PCB production, shows no fluorescence (Fig. 2a). One round of mutagenesis created a FP that covalently attached PCB (R1+PCB) and was fluorescent (Supplementary Fig. 2a) with one mutation, N42I. Round 2 produced a FP (R2-1+PCB) that was 27-fold brighter with mutations (Y65F, G66C) in the homodimeric interface. Round 3 produced R3-2+PCB (V83I, V98M) and was 1.7-fold brighter (Supplementary Fig. 2b). R2-1+PCB and R3-2+PCB have QYs of 7.2\% and 13\% and extinction coefficients $(\mathrm{EC})=65,000$ and $74,000 \mathrm{M}^{-1} \mathrm{~cm}^{-1}$, respectively, showing increased brightness $\left(\mathrm{EC}^{*} \mathrm{QY}\right)$ correlated with biophysical properties.

After PCB selections, the PcyA gene was removed, leaving only BV production. R2-1 and R3-2 lacked fluorescence with BV. Round 4 produced a FP that covalently attached BV and had red-shifted fluorescence (R4-1, lack of +PCB signifies +BV). R4-1 contained four mutations, of which three (G45S, R61H, Q129K) are necessary for BV covalent attachment. Round 5 selected R5-2 with two mutations (G4C, Y56H), which red-shifted excitation and emission by $\sim 49 \mathrm{~nm}$ (Supplementary Table 1 ) relative to R3-2+PCB and illustrates the 
malleability of wavelengths. R4-1 and R5-2 had QYs=9.0\% and 5.3\% and ECs=93,000 and 71,000, respectively, showing diminished QY with red-shifted fluorescence.

At Round 6, the selection was altered to create blue-shifted APCaFPs+BV and 5-10 bright colonies/plate were selected, mixed, grown in lysogeny broth (LB), and the mixture of DNA was purified and retransformed to evolve FPs that grew faster in Escherichia coli. R6-6 was selected with $650 \mathrm{~nm}$ excitation to blue-shift fluorescence. R6-6 had two mutations, F36L, D73G, and had a QY=9.6\% and EC=190,000, which were improved relative to R5-2. R7-7 contained three mutations (E18K, R33H, M131I). R8-8 and R8-9 contained three mutations (E48D, G96A, K118N) and (K9N, H33R, G96D), respectively. E48D and G96A/D are located near BV, while K9N, H33R, K118N alter surface charge. R8-8 and R8-9 had QYs of $13 \%$ and $12 \%$ and $E C s=260,000$ and 175,000 , respectively, which should have been less fluorescent than R7-7 (QY=16\%, EC=250,000). R7-7, R8-8, and R8-9 were respectively 1.3-, 5.8- and 5.7-fold brighter than R4-1 (Supplementary Fig. 2c). Clearly, fluorescence brightness is not strictly limited to QY*EC and is dependent on protein production/stability, which differs despite having the same Escherichia coli, arabinose, plasmid, promoter, amino acid length, number of bacteria (OD600), media, propagation temperature, and growth time. Round 9 FPs were not brighter than Round 8, so 20 clones were randomly mutagenized. R10-10 was 8.3-fold brighter than R4-1 and contained S15T, R33H, G96A mutations. $\mathrm{R} 10-10$ had a $\mathrm{QY}=15 \%$ and $\mathrm{EC}=200,000$, which was comparable to $\mathrm{R} 7-7$ (QY=16\% and $\mathrm{EC}=250,000$ ), but R10-10 and R7-7 are 8.3- and 1.3-fold, respectively, brighter than R4-1 (Supplementary Fig. 2d). R11-2 was slightly more fluorescent than R10-10 (Supplementary Fig. 2e), but lacked improvement in biophysical properties. R10-10 and Round 11 library were mutated for the twelfth, final selection, yielding smURFP with two mutations (Y59F, G82S) and C4S removed disulfide bond formation. APCaFPs are aligned in Supplementary Fig. 2f. SmURFP is $\sim 650$-fold brighter than $\mathrm{R} 1+\mathrm{PCB}$. BV (QY=0.013\%) shows extremely weak fluorescence, but covalent attachment to smURFP (QY=18\%), the QY is increased $\sim 1,400$-fold due to BV rigidification and has spectral properties similar to Cy5 (Fig. 2a,b, full absorbance in Supplementary Fig. 3a).

\section{Characterization of smURFP and tandem dimer smURFP}

SmURFP was compared to BPH FPs with identical growth conditions in Escherichia coli. Fluorescence of smURFP is greater than IFP1.4 and iRFP713 (Fig. 2c). Mass spectrometry (MS) revealed that BV attachment to APCaFPs/smURFP is limited and the predominant fluorescent species has 1 BV (Supplementary Fig. 3)

SmURFP ran as a $32 \mathrm{kD}$ homodimer on a native gel. R10-10 ran as a $32 \mathrm{kD}$ homodimer, while R4-1 was a tetramer (Supplementary Fig. 4a). Tandem dimer smURFP (TDsmURFP) was created by adding a 23 amino acid linker between subunits and had $\sim 70 \%$ fluorescence of smURFP in bacteria (Fig. 2c). TDsmURFP (33 kD) ran near smURFP. TdTomato (54 kD) and IFP1.4 (37 kD) confirmed the dimeric nature (Supplementary Fig. 4a,b). To confirm covalent BV attachment, the FPs were run on a denaturing gel and BV was detected with zinc $^{25}$. IFP1.4, smURFP, and TDsmURFP showed correct MW, with BV covalently attached (Supplementary Fig. 4c). APCaC52 is conserved and covalently attaches PCB (Supplementary Figs. 1,5a). The C52S mutation eliminates smURFP fluorescence 
(Supplementary Fig. 5b,c) and C52 remains the BV attachment site. The large EC of smURFP, $180,000 \mathrm{M}^{-1} \mathrm{~cm}^{-1} / \mathrm{BV}$ chromophore, and relatively large QY=18\% make smURFP biophysically as bright as eGFP. BPH FPs have low QYs and are dimmer than smURFP (Table 1).

MS showed that APCaFPs contain <1 BV per dimer when expressed with HO-1 in bacteria (Supplementary Fig. 3). Measurement of BV incorporation rate identified if this was due to lack of BV and/or smURFP's affinity for BV. SmURFP fluorescence requires BV binding and covalent attachment. $0.5 \mu \mathrm{M}$ smURFP ( $1 \mu \mathrm{M}$ empty chromophore sites) was mixed with $0.1,1$, or $10 \mu \mathrm{M}$ BV (Supplementary Fig. 6). Fluorescence approached its asymptotic level as an exponential growth in time with half-life $\sim 39$ min, independent of BV concentration, consistent with smURFP+BV forming a nonfluorescent high-affinity complex prior to development of fluorescence and covalent attachment to BV. Two-step kinetics are typical for phycobiliproteins and phytochromes ${ }^{26,27}$. Increasing pH increases $\mathrm{C} 52$ nucleophilicity and increases the rate of fluorescence development (Supplementary Table 2). The low stoichiometry of BV relative to smURFP reflected limiting levels of BV during protein expression.

\section{SmURFP expression in neurons}

SmURFP fluorescence was compared to the coral-derived red FP, mCherry, because fluorescence is spectrally separate from smURFP. Lentivirus was created with smURFP T2A mCherry, where T2A is a self-cleaving peptide sequence that ensures production of both FPs at a similar rate. Neuronal culture shows colocalized expression of both FPs. mCherry shows lysosomal aggregation ${ }^{28}$, which is not seen with smURFP (Supplementary Fig. 7).

\section{Increasing chromophore within mammalian cells}

SmURFP expresses better than BPH FPs in bacteria (Fig. 2c), which was hoped to be true in mammalian cells. SmURFP/TDsmURFP fluorescence is less than eGFP in HEK293A cells. We hypothesized that BV has low membrane permeability. $25 \mu \mathrm{M} \mathrm{BV} \mathrm{(3} \mathrm{h)} \mathrm{addition}$ increased smURFP and TDsmURFP fluorescence by 4.7- and 6.7-fold, respectively (Fig. 3a). Purification of FPs+BV in Escherichia coli requires HO-1 to produce BV and expression of HO-1 in mammalian cells should increase BV concentration. The production of heme is highly orchestrated (Supplementary Fig. 8a). Expression of HO-1 with smURFP/ TDsmURFP significantly increased fluorescence 2.8- and 2.0-fold, respectively. The expression of HO-1 with 5-aminolevulinic acid (5-ALA, fuel production of heme) and $\mathrm{FeSO}_{4}$ (to reduce the accumulation of fluorescent PPIX) showed significant increases in smURFP and TDsmURFP fluorescence of 7.7- and 7.0-fold, respectively (Fig. 3a). To prove the benefit of extra BV is not limited to smURFP, we expressed HO-1 with BPH FPs. IFP1.4, IFP2.0, and iRFP713 all showed significant increases in fluorescence of 7.1-, 8.2-, and 5.7-fold, respectively, with HO-1 + 5-ALA $+\mathrm{FeSO}_{4}$ even though iRFP713 is claimed not to require exogenous $\mathrm{BV}^{16}$ (Fig. 3b).

The more hydrophobic biliverdin dimethylester $\left(\mathrm{BVMe}_{2}\right.$, Fig. 1c) should have greater membrane permeability. The carboxylic acids of BV are recognition motifs and must be free for BPHs, while carboxylic groups of BV are exposed on smURFP and tolerate esterification 
(Fig. 3c,d). $25 \mu \mathrm{M} \mathrm{BV} \mathrm{(3} \mathrm{h)} \mathrm{significantly} \mathrm{increased} \mathrm{fluorescence} \mathrm{of} \mathrm{BPH} \mathrm{FPs} \mathrm{(Fig.} \mathrm{3e,}$ Supplementary Fig. 9). $25 \mu \mathrm{M} \mathrm{BVMe} 2 / \mathrm{PCB}(3 \mathrm{~h})$ greatly increased smURFP fluorescence by 18- and 10-fold, respectively, relative to $25 \mu \mathrm{M}$ BV (Fig. 3e). TDsmURFP was brightened by 4.0 - and 5.8-fold. $25 \mu \mathrm{M}$ PCB added to IFP2.0 blue-shifted and decreased fluorescence. IFP2 $.0+25 \mu \mathrm{M} \mathrm{BVMe}_{2}$ resulted in no fluorescence increase (Supplementary Fig. 11). If esterases removed methyl groups on $\mathrm{BVMe}_{2}, \mathrm{BV}$ would be formed and enhance the fluorescence of IFP2.0. SmURFP+BVMe 2 is $\geq 7$-fold brighter than IFP2.0/iRFP713 (Fig. $3 e)$. Concentration dependence of different chromophores was analyzed. PCB requires $\geq 40$ $\mu \mathrm{M}$ for fluorescence similar to $2.5 \mu \mathrm{M} \mathrm{BVMe} 2 . \mathrm{BVMe}_{2}$ penetrates the membrane best, which is saturated at $\mathcal{2} .5 \mu \mathrm{M}$. Comparing fluorescence to eGFP (expressed after an internal ribosomal entry site (IRES)), smURFP has 5-fold greater fluorescence with $\mathrm{BVMe}_{2}$ (Supplementary Fig. 11). Based on biophysical properties, smURFP is as bright as eGFP and eGFP expression is reduced by being 3' of the IRES. In mammalian cells, smURFP has the potential to be as bright as eGFP by increasing the concentration of chromophore in the cytoplasm.

BV contains two negatively charged carboxylic acids. Acidification will protonate the carboxylic acids and make BV membrane-permeant. $25 \mu \mathrm{M} \mathrm{BV}$ added at pH $6.4(3.5 \mathrm{~h})$ increased fluorescence 3-fold relative to $\mathrm{BV}$ at $\mathrm{pH}$ 7.5. As controls, $\mathrm{BVMe}_{2}$ incorporation or eGFP expression was not affected by a drop in extracellular $\mathrm{pH}$ from 7.5 to $6.4(3.5 \mathrm{~h})$, showing no change in protein stability, protein translation, and cell health. Protonation of $\mathrm{BV}$ increases membrane permeability, but fluorescence is 5-fold less than $\mathrm{BVMe}_{2}$.

\section{smURFP and TDsmURFP with different chromophores}

SmURFP and TDsmURFP have two chromophore sites, but the predominant species contains only 1 BV. SmURFP/TDsmURFP were expressed without HO-1 and purified without chromophore, then chromophores were added in vitro. Samples were verified with MS (Supplementary Fig. 12) and biophysical properties were measured. For BV and PCB, two chromophores could attach, but the second chromophore partially quenched fluorescence and reduced the QY (Supplementary Table 3). 2 PCB had a $40 \mathrm{~nm}$ red-shifted fluorescence (Supplementary Fig. 13g,i). A single $\mathrm{BVMe}_{2}$ bound to smURFP, while 2 $\mathrm{BVMe}_{2}$ bound to TDsmURFP (Supplementary Fig. 12f,1). For BVMe ${ }_{2}$, the QY=12\% remains constant. SmURFP/TDsmURFP+BVMe 2 have the brightest fluorescence not only because $\mathrm{BVMe}_{2}$ is freely membrane-permeant, but because the fluorescence is not quenched by excess chromophore.

\section{SmURFP in vivo}

HT1080 cells were transduced with lentivirus ( $₫ 99 \%$ efficiency, 2 d) (Supplementary Fig. 14a). $12.5 \mu \mathrm{M} \mathrm{BV}(3 \mathrm{~h}$ ) caused equivalent fluorescence of smURFP and mCherry in vitro (Supplementary Fig. 14b). SmURFP is expected to be $\sim 2$-fold brighter than mCherry and suffers from inadequate permeability of BV. HT1080 cells stably expressing smURFP and mCherry were injected into four mice bearing two tumor xenografts. SmURFP fluorescence was visible without exogenous BV (Fig. 4a), but fluorescent intensity was 35\% of mCherry (Supplementary Fig. 14c,d). $250 \mathrm{nmol} \mathrm{BV}$ was injected intravenously, but no fluorescent increase was seen after $2 \mathrm{~h}$. Fluorescence of mCherry was greatly attenuated through the 
skin with a loss of $74 \%$, while smURFP had a loss of only $25 \%$, illustrating the importance of using FR/NIR FPs for deep tissue imaging (Supplementary Fig. 14e).

SmURFP was compared to mCardinal ${ }^{29}$ in smaller tumors in an area with less blood flow (Supplementary Fig. 15). HT1080 cells were transduced with lentivirus expressing smURFP or mCardinal ( $₫ 95 \%$ efficiency, 2 weeks) (Supplementary Fig. 15a). The FPs were separated because of significant spectral overlap. SmURFP is 2-4-fold brighter than mCardinal when $\mathrm{BVMe}_{2}$ is added ( 2 days) (Supplementary Fig. $15 \mathrm{c}-\mathrm{e}$ ), but smURFP is much dimmer than mCardinal in vivo ( 7.3-fold at $607 \mathrm{~nm}, \sim 4$-fold at $640 \mathrm{~nm}$, Supplementary Fig. 15f). Injection of $250 \mathrm{nmol} \mathrm{BV} / \mathrm{BVMe}_{2}$ gives no increase in fluorescence in vivo, though $10 \mu \mathrm{M}$ $\mathrm{BV} / \mathrm{BVMe}_{2}$ added to excised tumors enhanced fluorescence and verifies smURFP expression (Supplementary Fig. 15g). $10 \mu \mathrm{M} \mathrm{BV/BVMe} 2$ added to plasma shows rapid removal of ester groups and slower degradation of impermeant BV (Supplementary Fig. 16), explaining the discrepancy.

\section{FP stability}

FP stability influences expression and fluorescent intensity. EGFP has a protein stability half-life $\left(t_{50 \%}\right)$ of $\sim 24 \mathrm{~h}^{30}$, while BPH FPs have $t_{50 \%}=\sim 4.4 \mathrm{~h}$ (Table 1 ). The fluorescence of smURFP in HEK293 cells continuously exposed to BV/BVMe 2 , but protein synthesis was halted by cycloheximide, declined with a $t_{50 \%}=33$ or $35 \mathrm{~h}$, respectively (Supplementary Fig. $17 \mathrm{a}, \mathrm{b})$. Shortening exposure of BV/BVMe $2(3 \mathrm{~h})$ showed little difference $\left(t_{50 \%}=30\right.$ and $33 \mathrm{~h}$, respectively) (Supplementary Fig. 17c,d). The lifetime of smURFP lacking chromophore was determined by addition of cycloheximide for various time points and $\mathrm{BVMe}_{2}$ was added $(1 \mathrm{~h})$. Apoprotein decayed with $t_{50 \%}=17 \mathrm{~h}$ (Supplementary Fig. 17e), showing a modest destabilization. As a control, eGFP had a $t_{50 \%}=21 \mathrm{~h}$, close to $24 \mathrm{~h}^{30}$ (Supplementary Fig. 17f). SmURFP initial mean fluorescence shows that membrane permeability is the major factor increasing fluorescence under nonsteady state conditions, but increasing incubation time results in increased protein stability and accumulation of smURFP+chromophore (Supplementary Fig. 17g).

\section{FP photostability}

SmURFP and TDsmURFP show greater photostability than eGFP, mCherry, IFP1.4, and Cy5 in vitro (Supplementary Fig. 18). The protein rather than the BV governs the photostability, because at an excitation intensity yielding 1,000 photons/s, IFP1.4+BV bleached with $t_{50 \%}=8.4 \mathrm{sec}$, while smURFP+BV had $t_{50 \%}=300 \mathrm{sec}$ (Table 1). Photostability was compared in cells. N/C-terminal smURFP fusions were created and showed correct cellular localization (Fig. 4). SmURFP mean $t_{50 \%}$ was 340 and $570 \mathrm{sec}$ with $\mathrm{BVMe}_{2}$ and BV, respectively, in mammalian cells (Supplementary Fig. 19). SmURFP+BV photostability is comparable to eGFP, but greater than mCherry and tdTomato (Supplementary Table 4).

\section{A FR/NIR fluorescent cell cycle biosensor}

We created a fluorescent biosensor at wavelengths longer than attainable with jellyfish/coralderived FPs using smURFP and IFP2.0 ${ }^{18}$. Miyawaki and co-workers ${ }^{4}$ pioneered the dynamic Fluorescent Ubiquitination-based Cell Cycle Indicator (FUCCI). Two spectrally distinct FPs are synthesized and degraded at opposing phases of the cell cycle. Typically a green FP is 
made during S/G2/M phase and degraded during late M/G1 phase, while an orange FP is made during G0/G1 phase and destroyed at the start of S phase ${ }^{4}$. SmURFP, TDsmURFP, and IFP2.0 were fused to hCdt1(30/120) and hGem(1/110) fragments containing ubiquitination sites for degradation ${ }^{4}$. Stable HEK293A cell lines were created. SmURFP fluorescence was greater than TDsmURFP. A stable cell line expressing mAG-hGem(1/110) and smURFPhCdt1(30/120) was created to verify proper function. SmURFP-hCdt1(30/120) shows proper temporal dynamics (Supplementary Fig. 20, Supplementary Video 1). Stably expressing IFP2.0-hGem(1/110) showed sufficient fluorescence, but not IFP2.0-hCdt1(30/120). Fluorescent time-lapse imaging verified that HEK293 cells stably expressing smURFPhCdt1(30/120) and IFP2.0-hGem(1/110) reciprocally lit up during the G0/G1 and S/G2/M phases, respectively (Fig. 5, Supplementary Video 2). FUCCI now works in the FR/NIR.

\section{DISCUSSION}

SmURFP (20 mutations) was evolved from TeAPCa to incorporate BV without a lyase, express efficiently with minimal toxicity, and fluoresce in an underrepresented wavelength range, excitation/emission of 640-650/660-670 nm. The screen was carried out in bacteria by selecting bright colonies with HO-1 coexpressed to supply BV. Variants that had higher $\mathrm{EC}^{*} \mathrm{QY}(\mathrm{R} 7-7)$ were passed over in favor of smURFP, which expressed at higher levels due to faster synthesis and/or slower degradation rates. smURFP was evolved to fluoresce like Cy5, but can be red-shifted by $48 \mathrm{~nm}$ (R5-2). There are thousands of APCa genes in cyanobacteria and red-algae, which gives this new class of FPs vast opportunities. Additionally, APC $\beta$ and other phycobilisome proteins (phycoerythrin/phycocyanin) could create additional FPs.

Comparison of BPH FPs and red FPs was performed using purified FPs from Escherichia coli, normalizing concentrations of FP with BV already covalently attached, and embedding FPs inside phantoms into mice ${ }^{16,17}$. This comparison does not accurately reflect FP expression and accessibility to BV in vivo. iRFP713 shows little fluorescence when compared mCardinal in myoblasts injected into mice ${ }^{29}$. SmURFP fluorescence is visible without exogenous BV (Fig. 4a) and may be advantageous for imaging cancer/maladies in vivo, where the production of hydrogen peroxide could alter immune system/inflammation response and/or alter disease progression ${ }^{9-12}$.

Membrane permeability of BV is a limiting factor for BPH FPs and smURFP (Fig. 3). The development of fluorescence is controlled by the covalent attachment of the cofactor, not by reversible noncovalent BV affinity. HO-1 expression in neurons increases IFP2.0 fluorescence $^{18}$. HO-1 increases fluorescence significantly for BPH and APCa FPs, and enhanced more with 5-ALA and Fe, which fuels production of heme that HO-1 converts to BV. SmURFP and TDsmURFP, unlike BPH FPs, tolerate the freely permeant $\mathrm{BVMe}_{2}$. This open chromophore-binding pocket (Fig. 3d) should allow for further modification of tetrapyrroles to modify not only membrane permeability, but also spectral and fluorescent properties.

FP photostability is essential for imaging extended time periods or superresolution. BPH FPs were originally nonfluorescent phototransducers that lacked evolutionary pressure to be light 
tolerant, whereas APCa is a component of the light harvesting phycobilisome, which is extremely tolerant of light. Without using special selections, smURFP is very photostable. Selection for increased photostability should enhance smURFP utility for superresolution applications.

The FR/NIR FUCCI creates a fluorescent biosensor with wavelengths inaccessible to jellyfish/coral FPs and does not produce hydrogen peroxide. Fluorescently monitoring the cell cycle has identified modifications to cell division, drug induced cell cycle modification $^{31}$, and quiescent cells ${ }^{32}$.

SmURFP is a new class of FP with a highly unnatural evolution including: 1) Covalently attach PCB (without an exogenous protein (lysase)) and fluoresce. 2) Covalently attach BV (first 3 rounds lacked BV fluorescence) and fluoresce. 3) Blue-shift BV fluorescence to match Cy5 and increase QY/brightness. and 4) Evolve highly stable FPs that do not inhibit growth. $\sim 10^{6}$ bacterial colonies were manually screened. SmURFP is biophysically the brightest FR/NIR FP created, fills a spectral gap in excitation wavelength, and chromophore attachment does not produce hydrogen peroxide. SmURFP is brighter than mCardinal in vitro when $\mathrm{BVMe}_{2}$ is added for 2 days, and comparable to eGFP in brightness, which is useful in cell culture for imaging low copy number proteins, superresolution imaging, and biosensors (FRET acceptor or red FP quencher). Despite its strong performance in vitro and modest visibility in vivo, smURFP is not ready for use in mice, for which screening new cofactors to achieve maximal brightness is required. SmURFP is the most photostable FP tested in the Tsien lab (all FPs tested by P. Steinbach $>10$ years $^{3}$ ). SmURFP is fusible to one of the most difficult proteins, a-tubulin (Fig. 4d). SmURFP-hCdt1(30/120) is functional (1 of 3 known FPs (mKO2, mCherry) despite the fact that jellyfish/coral-derived FPs mAG, eGFP, and mRFP1 are nonfunctional ${ }^{4}$. The FR/NIR FUCCI is the first biosensor using two BV-attaching FPs and can be used with traditional FUCCI to monitor the cell cycle of two cell types.

\section{ONLINE METHODS}

Chemicals

$\mathrm{BV}$ and $\mathrm{BVMe}_{2}$ were purchased from Frontier Scientific. PCB was extracted from Spirulina as described ${ }^{33}$.

\section{Gene synthesis, mutagenesis, and screening of libraries}

TeAPCa gene (codons optimized for Escherichia coli) was made by GenScript. Mutations were placed by random mutagenesis using GeneMorphII Random Mutagenesis Kit (Agilent Technologies). Screens for mutants that fluoresce with PCB or BV were performed by subcloning into a pBAD vector (Life Technologies) that expresses cyanobacteria Synechocystis HO-1 and Synechocystis PcyA genes for PCB production and Synechocystis $\mathrm{HO}-1$ only for BV production, as previously described ${ }^{34}$. Libraries were expressed in TOP10 Electrocompetent Escherichia coli cells (Life Technologies) with addition of $0.2 \%$ arabinose to LB agar plates (FPs were constitutively expressed in Escherichia coli). Fluorescence was imaged on plates using a BioSpectrum AC Imaging System (UVP) with 
$\mathrm{EX} / \mathrm{EM}=628(40) / 690(50) \mathrm{nm}$ for FPs+PCB, EX $/ \mathrm{EM}=685(40) / 710 \mathrm{LP} \mathrm{nm}$ for Rounds 4 and 5 FPs+BV, and EX / EM = 650(13) / 690(50) nm for Cy5-like FPs+BV (Rounds 612). At Round 6, 5-10 of the brightest colonies on each plate were combined in $\mathrm{LB}+0.2 \%$ arabinose, grown $\sim 18 \mathrm{~h}$, mixture of plasmid DNA was purified, TOP10 cells were transformed, and plated on LB/agar plates $+0.2 \%$ arabinose. Fluorescence was screened on plates (as described above) and 10-20 of the brightest colonies were screened in liquid culture fluorescence (Supplementary Fig. 2a-e), plasmid DNA purified, and DNA was sequenced. 2-3 of the brightest FPs plasmid DNA was chosen for mutagenesis for the subsequent round. When choosing DNA for subsequent mutagenesis rounds, genes with increased and/or novel silent DNA mutations were chosen over other DNA that had the same DNA and/or protein sequence of the previous round. Site-specific mutagenesis was performed using QuikChange Lightning Mutagenesis Kit (Agilent Technologies) to create smURFP (C4S mutation) and smURFP C52S.

\section{FP purification and fluorescent characterization in vitro}

FP genes were expressed on a $\mathrm{pBAD}$ vector (Life Technologies) with a polyhistidine tag on the C-terminus, cells were lysed with B-PER (Thermo Scientific), and purified using NiNTA (Qiagen) purification. PCB (HO-1+PcyA) and BV (HO-1) incorporation in Escherichia coli was performed simultaneously during constitutively active FP expression. Extinction coefficient was determined by PCB or BV absorbance as described ${ }^{35,36}$ (Table 1, smURFP +BV / TDsmURFP+BV and Supplementary Table 1) or calculated for the apoproteins by determining the protein concentration by the BCA protein assay (Pierce) and a specific chromophore concentration is added (specified in the Purification of FP lacking

chromophore and in vitro chromophore incorporation section) to get 1 and 2 chromophore species (Supplementary Table 3, not including (Escherichia coli)). When FP + chromophore (PCB or BV) are expressed in Escherichia coli, PCB and BV Q band has absorbance that overlaps with detection of BCA or Bradford assay of 562 and $592 \mathrm{~nm}$, respectively. We use the previously published protocols ${ }^{35,36}$, where there is no change of the $\mathrm{BV}$ absorbance at $390 \mathrm{~nm}$ when free in solution or attached to the FP. SmURFP+BV denaturation with $1 \mathrm{M}$ urea causes no change in the $\mathrm{BV}$ absorbance at $390 \mathrm{~nm}$, but the Q band is reduced $\sim 20$-fold. Therefore, the extinction coefficient of BV at $390 \mathrm{~nm}$ is used to calculate the concentration of holoprotein $(\mathrm{FP}+\mathrm{PCB} / \mathrm{BV})$ concentration.

EX and EM spectra were obtained using SPEX Fluorolog fluorometer (Horiba). Absorbance spectra were obtained using UV-VIS Spectrophotometer Cary Eclipse (Varian) or UV-2700 (Shimadzu). Quantum yield was determined relative to Cy5. Incorporation rates of BV on $0.5 \mu \mathrm{M}$ smURFP was performed by addition of smURFP to PBS alone or with $20 \%$ FBS and mixed by pipetting in a quartz cuvette. Chromophore was added last, mixed by pipetting, and fluorescence was recorded using SPEX Fluorolog fluorometer (Horiba). Photobleaching was characterized on bubbles of purified FP or Cy5 within mineral oil using light from a 150 $\mathrm{W}$ xenon arc lamp with $100 \%$ light through $\mathrm{EX} / \mathrm{EM}=628(40) / 680(30) \mathrm{nm}$ for smURFP, TDsmURFP, and Cy5 or EX / EM = 665(45) / 725(50) nm for IFP1.4 focused with a 40×, 1.2 numerical aperture C-Apochromat oil-immersion lens on a Zeiss Axiovert $200 \mathrm{M}$ inverted microscope. Photobleaching times were normalized to reflect an initial illumination 
intensity producing 1,000 photons per fluorescent molecule per sec as previously described $^{37}$.

\section{Purification of FP lacking chromophore and in vitro chromophore incorporation}

HO-1 was removed by digesting the pBAD vector (Life Technology) with MscI and PmeI (NEB). SmURFP and TDsmURFP was expressed and purified as described above. No chromophore was present on smURFP and TDsmURFP (confirmed by UV, fluorescent imaging, and MS). For predominant fluorescent species +2 chromophores, a 10-fold excess of chromophore was added to smURFP and TDsmURFP. For predominant fluorescent species +1 chromophore, a quarter concentration of chromophore was added to smURFP and TDsmURFP. Chromophore was added in PBS $+10 \%$ FBS at $37^{\circ} \mathrm{C}$ overnight. SmURFP/TDsmURFP+chromophore were purified using NiNTA (Qiagen) to remove excess/non-covalently attached chromophore.

\section{FP mass spectrometry}

FP mass was determined by liquid chromatography-mass spectrometry (LC-MS), where the protein column eluant was directly injected into the electrospray interface of an Orbitrap XL (Thermo Fisher). FP precipitation was minimized by using a steep gradient and injecting directly into the mass spectrometer. The $1100 \mathrm{LC}$ (Agilent Technologies) had solvent $\mathrm{A}=$ $2 \%$ acetonitrile (ACN), $0.1 \%$ formic acid (FA) and solvent $\mathrm{B}=90 \% \mathrm{ACN}, 0.1 \% \mathrm{FA}$, the flow rate was $80 \mu \mathrm{l} / \mathrm{min}$, and the gradient was $18 \%$ A to $100 \%$ B in 2.4 minutes. Protein was eluted within $10 \mathrm{~min}$ from a $1 \mathrm{~mm}$ inner diameter, $50 \mathrm{~mm}$ long PLRP-S C18 column (Agilent Technologies). Orbitrap IonSpray (electrospray) interface had a sheath gas flow rate of 34 and $\mathrm{T}=275{ }^{\circ} \mathrm{C}$. Capillary was $39 \mathrm{~V}$ and the tube lens was $140 \mathrm{~V}$. Full scan, mass spectra were collected in the ion trap and Fourier transform profile mode, with Orbitrap resolution 60,000, from 500 to 1,800 mass-to-charge units. Protein mass spectra were deconvolved using either the extract_msn program (Xcalibur software) or with ProMassCalc (ThermoFisher).

\section{Homology model creation, identifying dimeric interface, protein sequence alignment, and creating protein figures}

SmURFP homology model was created using Swiss-Model Server ${ }^{38}$ with P. yezoensis APCa crystal structure (1KN1.pdb ${ }^{39}$ and sequence in Supplementary Fig. 1). The homodimeric interface was identified using PatchDock ${ }^{40}$ and subsequently FireDock ${ }^{41}$. The lowest free energy structure is shown in Fig. 1d. Protein sequence alignments (Supplementary Figs. 1,2) were created using ClustalX ${ }^{42}$. All protein figures were created using UCSF Chimera package ${ }^{43}$.

\section{Construction of TDsmURFP bacterial expression vector}

TDsmURFP was created using the smURFP homology model (Fig. 1d) and approximating the distance from the $\mathrm{C}$-terminus to the $\mathrm{N}$-terminus of the second subunit. A 23 amino acid linker (GHGTGSTGSGSSGTASSEDNNMA) was sufficient and primers were created with 5' BamHI and 3' EcoRI restriction sites (IDT). SmURFP was PCR amplified with Phusion High-Fidelity DNA Polymerase (NEB) to create the right and left subunits using 23 amino 
acid linker primers. The two products were combined and TDsmURFP was created by bridging PCR with Phusion High-Fidelity DNA Polymerase (NEB). TDsmURFP was digested with BamHI-HF and EcoRI-HF (NEB), gel purified using Zymoclean Gel DNA Recovery Kit (Zymo), and subcloned into a pBAD (Life Technologies) vector containing HO-1 digested with BamHI-HF and EcoRI-HF (NEB) with T4 DNA Ligase (Life Technologies).

\section{Native PAGE, SDS denaturing PAGE, and Zinc blot}

Native PAGE was run using NativePAGE Novex Bis-Tris Gel System (Life Technologies) on NativePAGE 4-16\% Bis-Tris protein gels (Life Technologies). SDS denaturing PAGE was run using NuPAGE MOPS SDS running buffer (Life Technologies) and NuPAGE Novex 412\% Bis-Tris gels (Life Technologies). Precision Plus Protein Dual Color Standards (BioRad) were used as a MW ladder. Zinc blot was performed after running the SDS PAGE gel using the described method ${ }^{44}$. Fluorescence was imaged using a BioSpectrum AC Imaging System (UVP) with EX / EM = 535(45) / 605(70) nm for tdTomato, EX / EM = 650(13) / 690(50) $\mathrm{nm}$ for smURFP and TDsmURFP, EX / EM = 685(40) / 710LP nm for IFP1.4 and BV+zinc.

\section{Construction of lentiviral vectors, virus production, and neuron infection}

smURFP T2A mCherry, smURFP T2A mCardinal, smURFP, and mCardinal (Addgene 51311) were subcloned in a generation-two lentiviral vector with the CMV promoter for constitutive expression. Viruses were produced as described ${ }^{45}$. The procedures for extracting cultured neurons from rat pups were approved by the UCSD Institutional Animal Care and Use Committee (IACUC) (\#S03172R) and are consistent with the recommendations of the American Veterinary Medical Association. Primary cortical neurons were dissociated by papain from postnatal day 2 Sprague Dawley rats of either sex, plated on poly-D-lysine coated glass bottom culture dishes (MatTek, \#P35G-0-14-C), and cultured in Neurobasal A medium $+1 \times$ B27 supplement (Life Technologies) +2 mM GlutaMAX $^{\mathrm{TM}}$ (Life Technologies) $+1 \times$ Penicillin-Streptomycin (Fisher Scientific). Prior to fluorescent imaging, $25 \mu \mathrm{M}$ BV was added for $10 \mathrm{~min}$, removed, and washed with $2 \times 2 \mathrm{ml}$ Hank's Balanced Salt Solution (HBSS, Life Technologies) $+2 \mathrm{~g} / \mathrm{L}$ glucose $+20 \mathrm{mM}$ HEPES (pH 7.4), referred to has imaging solution. Fluorescent imaging was performed on in vitro day 15 after infection using a Zeiss Axiovert 200M inverted microscope controlled by SlideBook software. FPs were imaged as follows: smURFP/TDsmURFP EX / EM = 628(40) $/ 680(30) \mathrm{nm}$ and mCherry EX / EM = 580(20) / 653(95) nm.

\section{Fluorescence imaging of HT1080 tumor xenografts in vivo}

HT1080 cells were infected with CMV with smURFP T2A mCherry, smURFP T2A mCardinal, smURFP, and mCardinal lentiviruses in culture and expression was verified by fluorescence imaging (Supplementary Fig. 14a: $299 \%$ efficiency \& Supplementary Fig. 15a: $295 \%$ efficiency) using filters described in the previous section. All procedures using mice were approved by UCSD IACUC (\#S03172M) and are consistent with the recommendations of the American Veterinary Medical Association. One million HT1080 cells expressing smURFP T2A mCherry were injected subcutaneously into the lateral, ventral, upper right and left quadrant or smURFP and mCardinal were injected subcutaneously into the lateral, 
dorsal, lower right and left quadrant of four 5 week old athymic nude female mice. Tumors were allowed to grow until $\sim 0.1-1 \mathrm{~cm}$ in diameter ( 2 weeks). Mice were anesthetized with isoflurane (1.5\%) at a $2 \mathrm{~L} / \mathrm{min}$ flow, injected with ketamine/midazolam (IP, $80 \mathrm{mg} / \mathrm{kg}, 4 \mathrm{mg}$ / $\mathrm{kg}$ ), placed on a heated pad, and were imaged before exogenous BV injection. $250 \mathrm{nmol} \mathrm{BV}$ was injected by intravenous tail vein and mice were imaged 5, 30, 60, and 120 min after BV injection for smURFP T2A mCherry. $250 \mathrm{nmol} \mathrm{BV}$ or $\mathrm{BVMe}_{2}$ was injected by intravenous tail vein and mice were imaged $60 \mathrm{~min}, 24 \mathrm{~h}$, and $48 \mathrm{~h}$ after BV injection for smURFP and mCardinal tumors. Mice were imaged using a Maestro In Vivo Imaging System (CRI). FPs were imaged with the following filters: mCherry EX / EM $=590(23) / 615 \mathrm{LP} \mathrm{nm}$ and liquid crystal $=620 \mathrm{~nm}, \mathrm{mCardinal} \mathrm{EX} / \mathrm{EM}=607(36) / 645 \mathrm{LP} \mathrm{nm}$ and liquid crystal $=660 \mathrm{~nm}$, and smURFP EX $/ \mathrm{EM}=620(20) / 645 \mathrm{LP} \mathrm{nm}$ or EX $/ \mathrm{EM}=640(47) / 700 \mathrm{LP}$ and liquid crystal $=710 \mathrm{~nm}$. Image cubes were obtained and could be spectrally unmixed, but raw fluorescence images before chromophore injection are shown in Supplementary Fig. 14c and Supplementary Fig. $15 \mathrm{f}$ because no fluorescence increase was seen after $250 \mathrm{nmol} \mathrm{BV/}$ $\mathrm{BVMe}_{2}$ injection. Tumors were removed, washed $2 \times 2 \mathrm{ml}$ DMEM $+10 \% \mathrm{FBS}$, labeled in 2 $\mathrm{ml}$ DMEM $+10 \% \mathrm{FBS}+10 \mu \mathrm{M}$ BV/BVMe 2 at $4{ }^{\circ} \mathrm{C}$ for $24 \mathrm{~h}$, imaged, labeled in $2 \mathrm{ml}$ $\mathrm{DMEM}+10 \% \mathrm{FBS}+10 \mu \mathrm{M} \mathrm{BV} / \mathrm{BVMe} 2$ at $37^{\circ} \mathrm{C}$ for $24 \mathrm{~h}$, and imaged (Supplementary Fig. 15g).

\section{Plasma collection and $\mathrm{BV} / \mathrm{BVMe}_{2}$ concentration analysis}

Whole blood was collected via abdominal aorta and placed in tubes with lithium heparin (BD, 365971) on ice. Tubes were centrifuged to remove red bloods cells and plasma was stored on ice for $30 \mathrm{~min}$. Plasma that was clear was combined and aliquoted into $9 \times 70 \mu \mathrm{l}$ aliquots and placed on ice. The following 9 experiments were performed: starting plasma (1) and plasma $+10 \mu \mathrm{M} \mathrm{BV}(2-5)$ or $\mathrm{BVMe}_{2}(6-9)$ at time $=0$ at $4{ }^{\circ} \mathrm{C}$ and 15,60 , and 1,440 $\min$ at $37^{\circ} \mathrm{C}$. Reactions were stopped by freezing at $-80{ }^{\circ} \mathrm{C}$. Plasma samples were thawed, diluted $4 \times$ with cold $50 \% \mathrm{ACN}, 48 \%$ water $\left(\mathrm{H}_{2} \mathrm{O}\right)$, and $2 \%$ acetic acid, and centrifuged for 14 min at $4{ }^{\circ} \mathrm{C}$. Reverse phase HPLC was performed on an Agilent 1100 Series HPLC on a Phenomenex Luna C18(2) reverse phase column $100 \AA$, $250 \mathrm{~cm} \times 21.20 \mathrm{~mm}$ I.D. $10 \mu \mathrm{m}$ reverse phase column (00G-4253-P0 AX), with a $21 \mathrm{~min}, 10-90 \% \mathrm{H}_{2} \mathrm{O}$ :ACN (0.05\% TFA) gradient and a flow rate of $1 \mathrm{ml} / \mathrm{min}$ into a diode array detector and MSD-Ion Trap (Agilent LC/MSD trap XCT). $5 \mathrm{mM} \mathrm{BV/BVMe2} \mathrm{in} \mathrm{DMSO} \mathrm{was} \mathrm{diluted} \mathrm{and} \mathrm{used} \mathrm{to} \mathrm{calibrate} \mathrm{the}$ concentration of chromophore as a function of peak area (Supplementary Fig. 16).

\section{Mammalian expression plasmids, cell culture, transfection, chromophore addition, and fluorescent imaging}

SmURFP codons were optimized for human cell expression and the gene was created by GenScript. SmURFP, TDsmURFP (created from mammalian optimized smURFP), IFP1.4 (mammalian optimized from ${ }^{35}$ ), IFP2.0 (mammalian optimized from ${ }^{46}$ ), and iRFP713 (mammalian optimized from ${ }^{36}$ ) were PCR amplified using Phusion High-Fidelity DNA Polymerase (NEB) with primers containing 5' HindIII and 3' XhoI restriction enzyme sites. HindIII-HF and XhoI (NEB) digested PCR fragments were gel purified (Zymoclean Gel DNA Recovery Kit) and were ligated (T4 DNA Ligase, Life Technologies) into a similarly digested pCDNA3 IRES eGFP vector (bicistronic to express two FPs in the same cell). For creation of HO-1 expressing mammalian vectors, the Synechocystis HO-1 was used and the 
codons were not optimized for mammalian expression. Synechocystis HO-1 was directly amplified from the $\mathrm{pBAD}$ vector using primers containing 5' BsiWI and 3' XbaI restriction enzyme sites. PCDNA3 smURFP/TDsmURFP/IFP1.4/IFP2.0/iRFP713 IRES eGFP vectors were digested with BsiWI and XbaI (NEB), dephosphorylated (SAP, Roche), gel purified (Zymoclean Gel DNA Recovery Kit), and ligated (T4 DNA Ligase, Life Technologies) with similarly digested, purified HO-1 PCR fragment. For creation of smURFP fusions, smURFP was PCR amplified using Phusion High-Fidelity DNA Polymerase (NEB) with primers containing 5' AgeI and 3' NotI restriction enzyme sites for N-terminal fusions or with primers containing 5' AgeI and 3' BspEI restriction enzyme sites for C-terminal fusions. mGeos2-VEL-ManII-N-10 (Addgene 57551) and Dronpa-PDHA1-N-10 (Addgene 57292) vectors were digested with AgeI and NotI (NEB) and mCherry- aTubulin-C-18 (Addgene 55148) and tdTomato-LaminB1-10 (Addgene 58107) were digested with AgeI and BspEI, dephosphorylated (SAP, Roche), gel purified (Zymoclean Gel DNA Recovery Kit), and ligated (T4 DNA Ligase, Life Technologies) with similarly digested, purified smURFP PCR fragment. HEK293A, HT1080 (ATCC), and PC3 (ATCC) cells were grown in Dulbecco's Modified Eagle's medium (DMEM, Corning) supplemented with 10\% FBS (Atlanta Biologicals) $+1 \times$ Penicillin-Streptomycin (Fisher Scientific), which is referred to as growth media, on poly-D-lysine coated glass bottom culture dishes (MatTek, \#P35G-0-14-C). The HEK cell line is listed in the ICLAC and NCBI Biosample databases and is commonly misidentified with the HeLa cell line. For expression of exogenous fluorescent proteins, there is no problem if there is a contaminating cell line because no endogenous biology or therapeutic results are being determined. HEK293A cells were purchased from Invitrogen (Life Technologies) and all experiments are derived from the same expanded frozen stock without cell line authentication or mycoplasma detection. Cells were transfected using $2 \mu \mathrm{g}$ of circular DNA + $5 \mu \mathrm{l}$ Lipofectamine 2000 (Life Technologies) in $2 \mathrm{ml}$ Opti-MEM (Life Technologies) for $4-5 \mathrm{~h}$, transfection media was discarded, and pre-warmed growth media was added. Chromophores ( $5 \mathrm{mM}$ in DMSO) were added at indicated concentrations to growth media, warmed for $10-15 \mathrm{~min}$ at $37^{\circ} \mathrm{C}$, and added to cells. For multiple time points, incubations were staggered to maintain equivalent incubation. Prior to imaging, cells were washed $1 \times 2 \mathrm{ml}$ growth media, $2 \times 2 \mathrm{ml}$ imaging solution, and imaged in $2 \mathrm{ml}$ imaging solution. Fluorescent imaging was performed on a Zeiss Axiovert 200M inverted microscope controlled by SlideBook software. FPs were imaged with the following settings: eGFP EX / $\mathrm{EM}=495(10) / 535(25) \mathrm{nm}$, tdTomato $\mathrm{EX} / \mathrm{EM}=540(25) / 595(50) \mathrm{nm}$, mCherry and mCardinal EX / EM = 580(20) $/$ 653(95) nm, smURFP and TDsmURFP EX $/ \mathrm{EX}=$ 628(40) / 680(30) nm, and IFP1.4, IFP2.0, and iRFP713 EX / EM = 665(45) / 725(50) nm. When comparing mean fluorescent intensity of FPs with different wavelengths, data was normalized to reflect chromophore absorbance, chromophore extinction coefficient, light power (measured with an IL1700 research radiometer (International Light Technologies)), objective, EX / EM filters transmission, and dichroic reflectance as described ${ }^{37}$.

\section{FP stability in HEK293A cells}

HEK293A cells were transfected with pCDNA3 smURFP IRES eGFP and incubated for 48 h. $25 \mu \mathrm{M} \mathrm{BV}$ or $5 \mu \mathrm{M} \mathrm{BVMe} 2$ was added to HEK293A cells as listed in Supplementary Fig. 17. $50 \mu \mathrm{g} / \mathrm{ml}$ cycloheximide $+25 \mu \mathrm{M} \mathrm{BV}, 5 \mu \mathrm{M} \mathrm{BVMe}_{2}$, or no chromophore was added and cells were imaged after 1, 10, 30, 60, 120, and $225 \mathrm{~min}$. Fluorescence was fit to a single 
exponential decay $\left(\mathrm{F}=\mathrm{A}\left[\exp ^{-\mathrm{kt}}\right]\right)$. Fitted values and $t_{50 \%}$ of FPs are listed in

Supplementary Fig. 17.

\section{Photobleaching FP or FP fusions in mammalian cells}

PC 3 cells were transfected with $2 \mu \mathrm{g}$ of circular DNA $+5 \mu$ Lipofectamine 2000 (Life

Technologies) in $2 \mathrm{ml}$ Opti-MEM (Life Technologies) for 4-5 h, transfection media was discarded, pre-warmed growth media was added, and protein was produced for $48 \mathrm{~h} .25 \mu \mathrm{M}$

$\mathrm{BV} / 1 \mu \mathrm{M} \mathrm{BVMe}_{2}$ was incubated for $4 \mathrm{~h}$. Cells were photobleached with continuous light exposure from a $150 \mathrm{~W}$ xenon arc lamp with eGFP EX / EM = 495(10) / 535(25) nm, tdTomato EX / EM = 540(25) / 595(50) nm, mCherry EX / EM = 580(20) / 653(95) nm, and smURFP EX / EM = 628(40) / 680(30) nm focused with a 40×, 1.2 numerical aperture CApochromat oil-immersion lens on a Zeiss Axiovert 200M inverted microscope. Photobleaching times were normalized to reflect an initial illumination intensity producing 1,000 photons per fluorescent molecule per sec as previously described ${ }^{37}$. Data was fit to a single exponential decay $\left(\mathrm{F}=\mathrm{A} \exp ^{-\mathrm{kt}}\right)$ or a double exponential decay $\left(\mathrm{F}=\mathrm{A}_{1} \exp ^{-\mathrm{k}} \mathrm{t}^{\mathrm{t}}+\mathrm{A}_{2}\right.$ $\exp ^{-k_{2} t}$ ) and data is shown in Supplementary Table 4.

\section{Creating and imaging transiently/stably expressing FR/NIR FUCCI HEK293A cells}

PCSII-EF containing mAG-hGem(1/110) or mKO2-hCdtI(30/120) (A. Miyawaki, RIKEN) were PCR amplified using Phusion High-Fidelity DNA Polymerase (NEB) with primers containing 5' HindIII-HF and 3' XbaI restriction enzyme sites. HindIII-HF and XbaI (NEB) digested PCR fragments were gel purified (Zymoclean Gel DNA Recovery Kit) and were ligated (T4 DNA Ligase, Life Technologies) into a similarly digested pCDNA3 vector, creating initial pCDNA3 mAG-hGem(1/110) or mKO2-hCdtI(30/120) vectors. SmURFP, TDsmURFP, and IFP2.0 were PCR amplified using Phusion High-Fidelity DNA Polymerase (NEB) with primers containing 5' HindIII and 3' XhoI, HindIII-HF and XhoI digested (NEB), gel purified (Zymoclean Gel DNA Recovery Kit). PCDNA3 mAG-hGem(1/110) or mKO2-hCdtI(30/120) were digested with HindIII-HF and XhoI (NEB) to remove the FP and linker, dephosphorylated (SAP, Roche), and gel purified (Zymoclean Gel DNA Recovery Kit). PCDNA3 X-hGem(1/110)/hCdtI(30/120) were ligated with smURFP, TDsmURFP, and IFP2.0 to create pCDNA3 smURFP/TDsmURFP/IFP2.0-hGem(1/110)/hCdtI(30/120), 6 plasmids with hygromycin $\mathrm{B}$ resistance to create stable cell lines.

HEK293A cells were transfected with Lipofectamine 2000 (Life Technologies) on glass bottom dishes (grown and transfected as described above). Initially, all 8 constructs (including mAG-hGem(1/110) and $\mathrm{mKO} 2-\mathrm{hCdtI}(30 / 120)$ ) were fluorescently imaged using a Zeiss Axiovert 200M inverted microscope controlled by SlideBook software. FPs were imaged as follows: $\mathrm{mAG} \mathrm{EX} \mathrm{/} \mathrm{EM}=495(10) / 535(25) \mathrm{nm}, \mathrm{mKO} 2 \mathrm{EX} / \mathrm{EM}=540(25) /$ 595(50) nm, smURFP/TDsmURFP EX / EX = 628(40) $/ 680(30) \mathrm{nm}$, and IFP2.0 EX / EM = 665(45) / 725(50) nm. smURFP/TDsmURFP/IFP2.0-hGem(1/110)/hCdtI(30/120) all had fluorescent nuclei when transiently expressed in HEK293A cells. A kill curve was generated with hygromycin B (Life Technologies) and $300 \mu \mathrm{g} / \mathrm{ml}$ hygromycin B was sufficient to kill untransfected HEK293A cells. HEK293A cells expressing mAG/IFP2.0-hGem(1/110) or mKO2/IFP2.0-hCdtI(30/120) were grown for 3 weeks in growth media $+300 \mu \mathrm{g} / \mathrm{ml}$ hygromycin B. Cells were selected using fluorescently activated cell sorting (FACS) using 
FACSDiVa (BD Biosciences) to give an enriched stable population containing the expressed FP-fusion. Cells expressing FPs were sorted using the following filters: $\mathrm{mAG}$ EX / EM = $488 / 535(20) \mathrm{nm}, \mathrm{mKO} 2 \mathrm{EX} / \mathrm{EM}=568 / 610(40) \mathrm{nm}$, and IFP2.0 EX / EM = 670 / 710LP $\mathrm{nm}$. HEK293A cells expressing mAG/IFP2.0-hGem(1/110) were transfected with smURFP/ TDsmURFP-hCdtI(30/120) or mKO2/IFP2.0-hCdtI(30/120) were transfected with smURFP/ TDsmURFP-hGem(1/110) and grown for an additional 3 weeks in growth media +300 $\mu \mathrm{g} / \mathrm{ml}$ hygromycin B. 198 individual clones were FACS (smURFP EX / EM = 647(10) / $675(20) \mathrm{nm}$ ) for the 8 combinations and grown for 2 weeks. Clones were initially assayed to verify both FPs and then proper growth (many cells show no growth and/or decreased cell cycle progression, which was typically accompanied by higher FP-fusion expression). TDsmURFP fusions were dimmer than smURFP fusions and were not characterized further. IFP2.0-hCdtI(30/120) or smURFP-hGem(1/110) fluorescence was lacking and/or extremely dim and could not be visualized with smURFP-hGem(1/110) or mKO2-hCdtI(30/120), respectively. mAG-hGem(1/110) + smURFP-hCdtI(30/120) and IFP2.0-hGem(1/110) + smURFP-hCdtI(30/120) (FR/NIR FUCCI) had sufficient fluorescence and 3 clones of each were grown and re-analyzed for fluorescence of both FPs and adequate growth. A single clone of each was imaged by time-lapse fluorescence microscopy.

\section{Time-lapse imaging of FUCCI HEK293A cells}

HEK293A cells stably expressing FUCCI were grown in growth media on glass bottom dishes coated with poly-D-lysine. After 2 days, media was changed to $2 \mathrm{ml}$ Leibovitz L-15 medium (no phenol red, Life Technologies) $+1 \mathrm{~g} / \mathrm{L}$ glucose $+200 \mu \mathrm{M}$ Trolox $+200 \mu \mathrm{M} \mathrm{L}-$ ascorbic acid $+12.5 \mu \mathrm{M}$ BV for 1 day. A Zeiss Axiovert 200M inverted microscope with temperature control chamber was allowed to equilibrate at $37^{\circ} \mathrm{C}$ for $\sim 30$ min and HEK293A FUCCI cell imaging dish was placed on the stage. The plastic lid was removed and $2.2 \mathrm{ml}$ of mineral oil was added to the top (ensuring complete seal to avoid media evaporation). A metal imaging dish weight sealed with a glass cover slip was placed on top of the imaging chamber to avoid focus drift during imaging. The microscope + dish was equilibrated for $\sim 1$ $\mathrm{h}$ at $37^{\circ} \mathrm{C}$. Cells were imaged every $15 \mathrm{~min}$, using a $10 \%$ neutral density filter. FPs were imaged as follows: $\mathrm{mAG}$ EX $/ \mathrm{EM}=488 / 535(20) \mathrm{nm}$, smURFP-hCdtI(30/120) EX $/ \mathrm{EM}=$ $628(40) / 680(30) \mathrm{nm}$, and IFP2.0-hGem(1/110) EX / EM = 665(45) $/ 725(50) \mathrm{nm}$. Movies were created in ImageJ ${ }^{47}$. All 3 image channels (DIC, smURFP-hCdtI(30/120), and mAG/ IFP2.0-hGem(1/110)) were opened separately as a hyperstack and brightness/contrast were adjusted as desired. The time was added to the DIC stack using Time Stamper (ImageJ plugin) and the 3 channels were merged using the following pseudocoloring: red; smURFPhCdtI(30/120), green; mAG/IFP2.0-hGem(1/110), and gray; DIC. The AVI movie was exported using JPEG compression and 10 frames per second in ImageJ.

\section{Experimental setup, data analysis, and statistical methods}

For cell culture experiments, sample size of $\geq n=30$ cells ( $n$ listed with each experiment) were chosen to ensure $P$ values $<0.03$ for the majority of significant comparisons. For animal experiments, 4 mice with 2 tumors each ( $n=8$ tumors) was chosen arbitrarily to ensure reproducibility of results. No cell culture dishes or animals were excluded from analysis for any reason. For animal studies, no randomization or blinding was utilized because both mCherry and smURFP FPs were simultaneously expressed on a bicistronic 
mRNA in each tumor cell that made up the tumor or smURFP and mCardinal tumors were imaged simultaneously on the same mouse. Tumors were imaged with both sets of excitation/emission filters. Fluorescent images were adjusted and analyzed using Image ${ }^{47}$. Graphs, statistics, and statistical significance tests were generated using KaleidaGraph 4.1 (Synergy). Comparisons were performed on mean fluorescent intensity with a one-way ANOVA with significance level set at $\alpha=0.05$. Equivalence of variance was determined for the one-way ANOVA with a post hoc test of Tukey honestly significant difference (HSD) for comparisons between any two samples. All error bars are standard error of the mean (s.e.m), except Fig. 3a,b is calculated as error propagation of the s.e.m..

\section{DNA constructs}

GenBank/EMBL/DDBJ: smURFP, KX449134; TDsmURFP, KX449135. Bacterial and mammalian expression plasmids and lentiviral transfer vectors are available at Addgene: smURFP, 80341, 80343, 80345, 80347, 80348, 80349; and TDsmURFP, 80342, 80344, 80346.

\section{Supplementary Material}

Refer to Web version on PubMed Central for supplementary material.

\section{Acknowledgments}

National Institute of General Medical Sciences Postdoctoral Fellowship F32GM089114 supported the project (E.A.R.). US National Institutes of Health grants GM086197 and NS027177 and the Howard Hughes Medical Institute (R.Y.T.) supported the project. We thank P. Steinbach for measuring in vitro photostability, Q. Xiong for FACS, S. Adams for advice and experimental expertise, and P. Arcaira for help with mouse experiments. We thank M. Lin (Departments of Pediatrics and Bioengineering, Stanford, Stanford, CA) for iRFP713 vectors, M. Davidson (Addgene) for fusion vectors, and A. Miyawaki (Laboratory for Cell Function Dynamics, RIKEN Brain Science Institute, Wako, Japan) for FUCCI vectors.

\section{References}

1. Giepmans BN, Adams SR, Ellisman MH, Tsien RY. The fluorescent toolbox for assessing protein location and function. Science. 2006; 312:217-224. [PubMed: 16614209]

2. Tsien RY. Constructing and exploiting the fluorescent protein paintbox (Nobel Lecture). Angew. Chem. Int. Ed. Engl. 2009; 48:5612-5626. [PubMed: 19565590]

3. Shaner NC, Steinbach PA, Tsien RY. A guide to choosing fluorescent proteins. Nat. Methods. 2005; 2:905-909. [PubMed: 16299475]

4. Sakaue-Sawano A, et al. Visualizing spatiotemporal dynamics of multicellular cell-cycle progression. Cell. 2008; 132:487-498. [PubMed: 18267078]

5. Tsien RY. The green fluorescent protein. Annu. Rev. Biochem. 1998; 67:509-544. [PubMed: 9759496]

6. Shaner NC, et al. Improved monomeric red, orange and yellow fluorescent proteins derived from Discosoma sp. red fluorescent protein. Nat. Biotechnol. 2004; 22:1567-1572. [PubMed: 15558047]

7. Moore MM, Oteng-Pabi SK, Pandelieva AT, Mayo SL, Chica RA. Recovery of red fluorescent protein chromophore maturation deficiency through rational design. PLoS One. 2012; 7:e52463. [PubMed: 23285050]

8. Tubbs JL, Tainer JA, Getzoff ED. Crystallographic structures of Discosoma red fluorescent protein with immature and mature chromophores: linking peptide bond trans-cis isomerization and acylimine formation in chromophore maturation. Biochemistry. 2005; 44:9833-9840. [PubMed: 16026155] 
9. Veal EA, Day AM, Morgan BA. Hydrogen peroxide sensing and signaling. Mol. Cell. 2007; 26:114. [PubMed: 17434122]

10. Hussain SP, Hofseth LJ, Harris CC. Radical causes of cancer. Nat. Rev. Cancer. 2003; 3:276-285. [PubMed: 12671666]

11. Weitzman SA, Gordon LI. Inflammation and cancer: role of phagocyte-generated oxidants in carcinogenesis. Blood. 1990; 76:655-663. [PubMed: 2200535]

12. Barnham KJ, Masters CL, Bush AI. Neurodegenerative diseases and oxidative stress. Nat. Rev. Drug Discov. 2004; 3:205-214. [PubMed: 15031734]

13. Kumagai A, et al. A bilirubin-inducible fluorescent protein from eel muscle. Cell. 2013; 153:16021611. [PubMed: 23768684]

14. Konig K. Multiphoton microscopy in life sciences. J. Microsc. 2000; 200:83-104. [PubMed: 11106949]

15. Shu X, et al. Mammalian expression of infrared fluorescent proteins engineered from a bacterial phytochrome. Science. 2009; 324:804-807. [PubMed: 19423828]

16. Filonov GS, et al. Bright and stable near-infrared fluorescent protein for in vivo imaging. Nat. Biotechnol. 2011; 29:757-761. [PubMed: 21765402]

17. Shcherbakova DM, Verkhusha VV. Near-infrared fluorescent proteins for multicolor in vivo imaging. Nat. Methods. 2013; 10:751-754. [PubMed: 23770755]

18. Yu D, et al. An improved monomeric infrared fluorescent protein for neuronal and tumour brain imaging. Nat. Commun. 2014; 5:3626. [PubMed: 24832154]

19. Auldridge ME, Satyshur KA, Anstrom DM, Forest KT. Structure-guided engineering enhances a phytochrome-based infrared fluorescent protein. J. Biol. Chem. 2012; 287:7000-7009. [PubMed: 22210774]

20. Fischer AJ, Lagarias JC. Harnessing phytochrome's glowing potential. Proc. Natl. Acad. Sci. U S A. 2004; 101:17334-17339. [PubMed: 15548612]

21. Yeh SW, Ong LJ, Clark JH, Glazer AN. Fluorescence properties of allophycocyanin and a crosslinked allophycocyanin trimer. Cytometry. 1987; 8:91-95. [PubMed: 3100257]

22. Tooley AJ, Cai YA, Glazer AN. Biosynthesis of a fluorescent cyanobacterial C-phycocyanin holoa subunit in a heterologous host. Proc. Natl. Acad. Sci. U S A. 2001; 98:10560-10565. [PubMed: 11553806]

23. Zhang J, et al. Fused-gene approach to photoswitchable and fluorescent biliproteins. Angew. Chem. Int. Ed. Engl. 2010; 49:5456-5458. [PubMed: 20583017]

24. Harris, JW.; Kellermeyer, RW. The Red Cell, Edn. Rev. Cambridge, Mass: Harvard University Press; 1970.

25. Wahleithner JA, Li LM, Lagarias JC. Expression and assembly of spectrally active recombinant holophytochrome. Proc. Natl. Acad. Sci. U S A. 1991; 88:10387-10391. [PubMed: 1961704]

26. Arciero DM, Bryant DA, Glazer AN. In vitro attachment of bilins to apophycocyanin. I. Specific covalent adduct formation at cysteinyl residues involved in phycocyanobilin binding in Cphycocyanin. J. Biol. Chem. 1988; 263:18343-18349. [PubMed: 3142876]

27. Li L, Murphy JT, Lagarias JC. Continuous fluorescence assay of phytochrome assembly in vitro. Biochemistry. 1995; 34:7923-7930. [PubMed: 7794904]

28. Katayama H, Yamamoto A, Mizushima N, Yoshimori T, Miyawaki A. GFP-like proteins stably accumulate in lysosomes. Cell Struct. Funct. 2008; 33:1-12. [PubMed: 18256512]

29. Chu J, et al. Non-invasive intravital imaging of cellular differentiation with a bright red-excitable fluorescent protein. Nat. Methods. 2014; 11:572-d578. [PubMed: 24633408]

30. Stack JH, Whitney M, Rodems SM, Pollok BA. A ubiquitin-based tagging system for controlled modulation of protein stability. Nat. Biotechnol. 2000; 18:1298-1302. [PubMed: 11101811]

31. Sakaue-Sawano A, Kobayashi T, Ohtawa K, Miyawaki A. Drug-induced cell cycle modulation leading to cell-cycle arrest, nuclear mis-segregation, or endoreplication. BMC Cell Biol. 2011; 12:2. [PubMed: 21226962]

32. Tomura M, et al. Contrasting quiescent G0 phase with mitotic cell cycling in the mouse immune system. PLoS One. 2013; 8:e73801. [PubMed: 24066072] 


\section{References}

33. Toettcher JE, Gong D, Lim WA, Weiner OD. Light control of plasma membrane recruitment using the Phy-PIF system. Methods Enzymol. 2011; 497:409-423. [PubMed: 21601096]

34. Gambetta GA, Lagarias JC. Genetic engineering of phytochrome biosynthesis in bacteria. Proc. Natl. Acad. Sci. U S A. 2001; 98:10566-10571. [PubMed: 11553807]

35. Shu X, et al. Mammalian expression of infrared fluorescent proteins engineered from a bacterial phytochrome. Science. 2009; 324:804-807. [PubMed: 19423828]

36. Filonov GS, et al. Bright and stable near-infrared fluorescent protein for in vivo imaging. Nat. Biotechnol. 2011; 29:757-761. [PubMed: 21765402]

37. Shaner NC, Steinbach PA, Tsien RY. A guide to choosing fluorescent proteins. Nat. Methods. 2005; 2:905-909. [PubMed: 16299475]

38. Arnold K, Bordoli L, Kopp J, Schwede T. The SWISS-MODEL workspace: a web-based environment for protein structure homology modelling. Bioinformatics. 2006; 22:195-201. [PubMed: 16301204]

39. Liu JY, Jiang T, Zhang JP, Liang DC. Crystal structure of allophycocyanin from red algae Porphyra yezoensis at 2.2-A resolution. J. Biol. Chem. 1999; 274:16945-16952. [PubMed: 10358042]

40. Schneidman-Duhovny D, Inbar Y, Nussinov R, Wolfson HJ. PatchDock and SymmDock: servers for rigid and symmetric docking. Nucleic Acids Res. 2005; 33:W363-W367. [PubMed: 15980490]

41. Andrusier N, Nussinov R, Wolfson HJ. FireDock: fast interaction refinement in molecular docking. Proteins. 2007; 69:139-159. [PubMed: 17598144]

42. Larkin MA, et al. Clustal W and Clustal X version 2.0. Bioinformatics. 2007; 23:2947-2948. [PubMed: 17846036]

43. Pettersen EF, et al. UCSF Chimera--a visualization system for exploratory research and analysis. J. Comput. Chem. 2004; 25:1605-1612. [PubMed: 15264254]

44. Wahleithner JA, Li LM, Lagarias JC. Expression and assembly of spectrally active recombinant holophytochrome. Proc. Natl. Acad. Sci. U S A. 1991; 88:10387-10391. [PubMed: 1961704]

45. Lin JY, Knutsen PM, Muller A, Kleinfeld D, Tsien RY. ReaChR: a red-shifted variant of channelrhodopsin enables deep transcranial optogenetic excitation. Nat. Neurosci. 2013; 16:1499_ 1508. [PubMed: 23995068]

46. Yu D, et al. An improved monomeric infrared fluorescent protein for neuronal and tumour brain imaging. Nat. Commun. 2014; 5:3626. [PubMed: 24832154]

47. Schneider CA, Rasband WS, Eliceiri KW. NIH Image to ImageJ: 25 years of image analysis. Nat. Methods. 2012; 9:671-675. [PubMed: 22930834] 

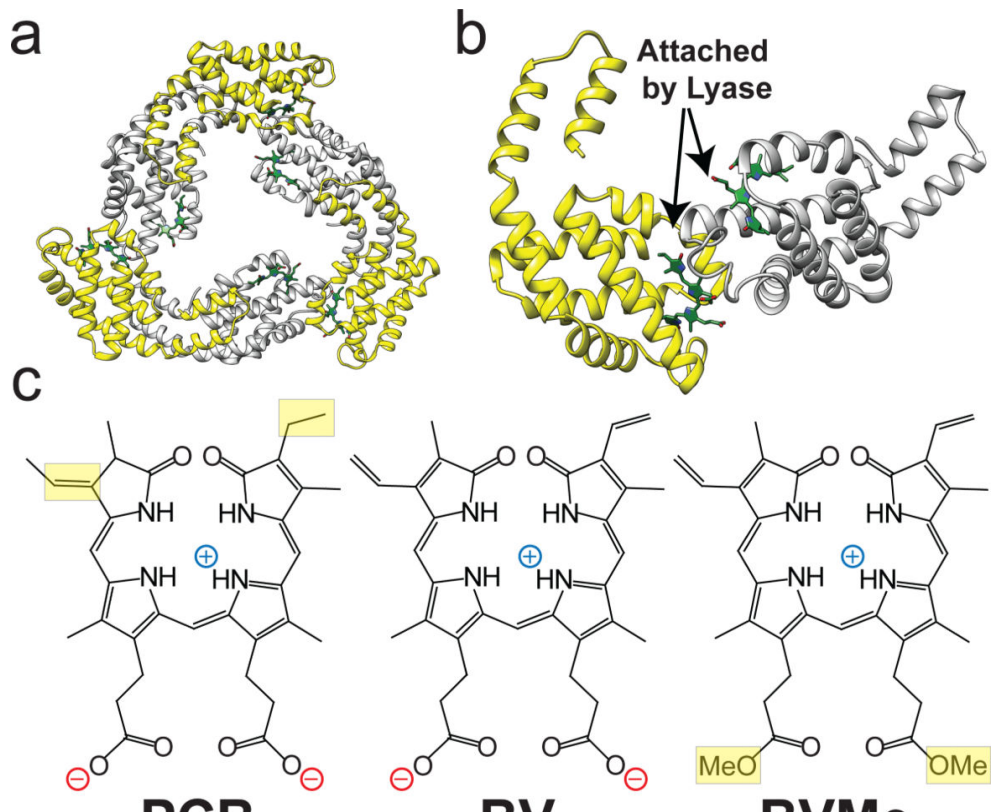

PCB

BV

$\mathrm{BVMe}_{2}$

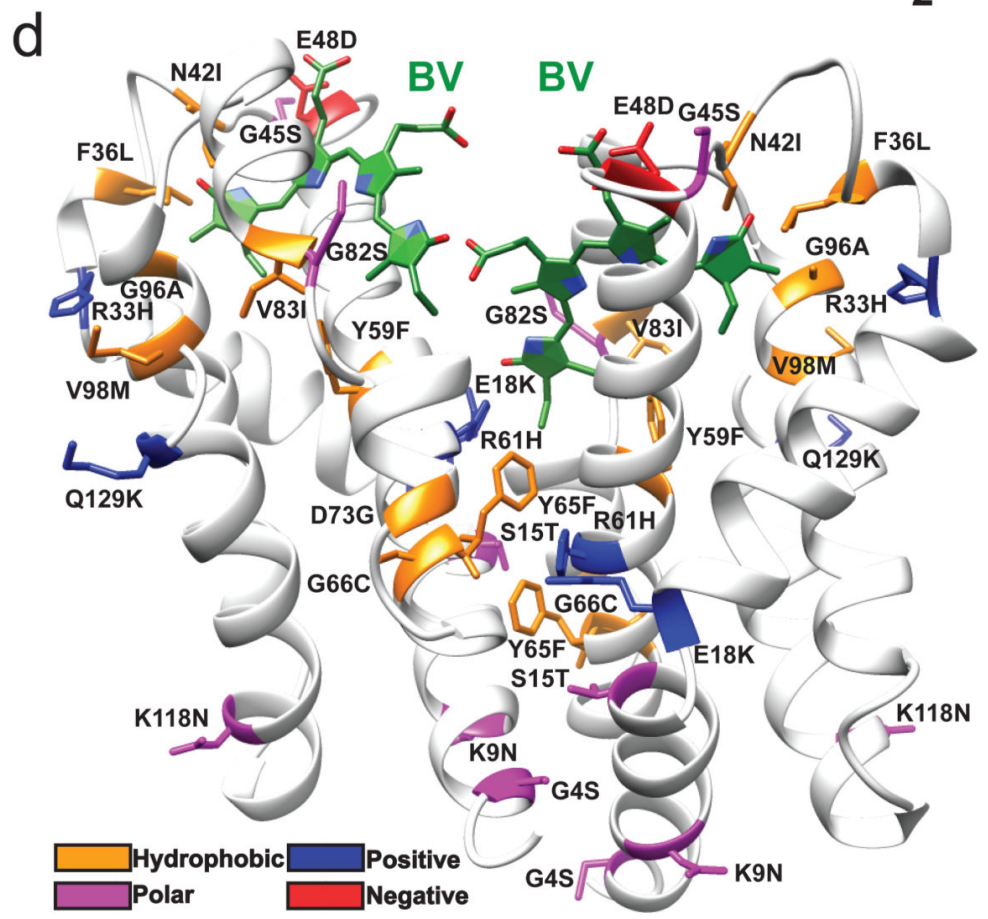

Figure 1.

Allophycocyanin, chromophore structures, and smURFP mutations. (a) Hexameric structure of APC from the phycobilisome (1ALL.pdb) composed of $3(a+\beta)$ dimers. Yellow is $a$, white is $\beta$, and green is PCB. (b) Enlarged $a+\beta$ dimer illustrating 2 unique PCB molecules (green) covalently attached by an external protein, known as a lyase. (c) Chromophores used in this study: $\mathrm{PCB}, \mathrm{BV}$, and $\mathrm{BVMe}_{2}$. Differences from BV are highlighted in yellow. (d) Homology model of the smURFP homodimer with 20 amino acids mutations highlighted. BV (green) covalent attachment is autocatalytic. 

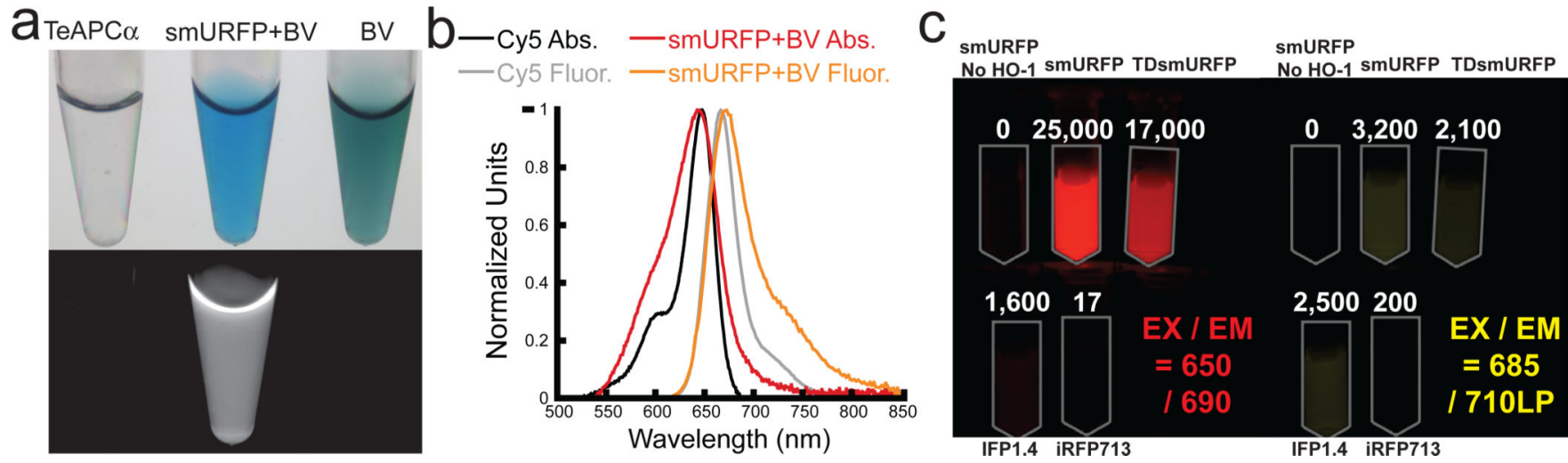

Figure 2.

smURFP+BV purified protein, spectra, comparing APCa and BPH FPs expressed in Escherichia coli, and smURFP+BV expressed in vivo. (a) Comparison of TeAPCa (expressed with PCB, but needs lyase for incorporation), smURFP+BV, and BV. Top is white light and bottom is fluorescence (EX / EM = $650 / 690 \mathrm{~nm})$. (b) Normalized absorbance and fluorescence spectra of Cy5 and smURFP+BV. (c) Comparison of APCa and BPH FPs expressed in Escherichia coli. Escherichia coli was grown in LB $+0.02 \%$ arabinose at $37{ }^{\circ} \mathrm{C}$ for $17.5 \mathrm{~h}$ and $2 \mathrm{ml}$ of culture was resuspended in $1 \mathrm{ml}$ PBS. Left \& Right are fluorescent images of FPs expressed in Escherichia coli $+\mathrm{HO}-1$, unless noted, and tubes are outlined in gray. Numbers in white are mean fluorescent intensity. Abs. is absorbance; Fluor. is fluorescence; EX is excitation maximum; EM is emission maximum; and LP is long pass. 


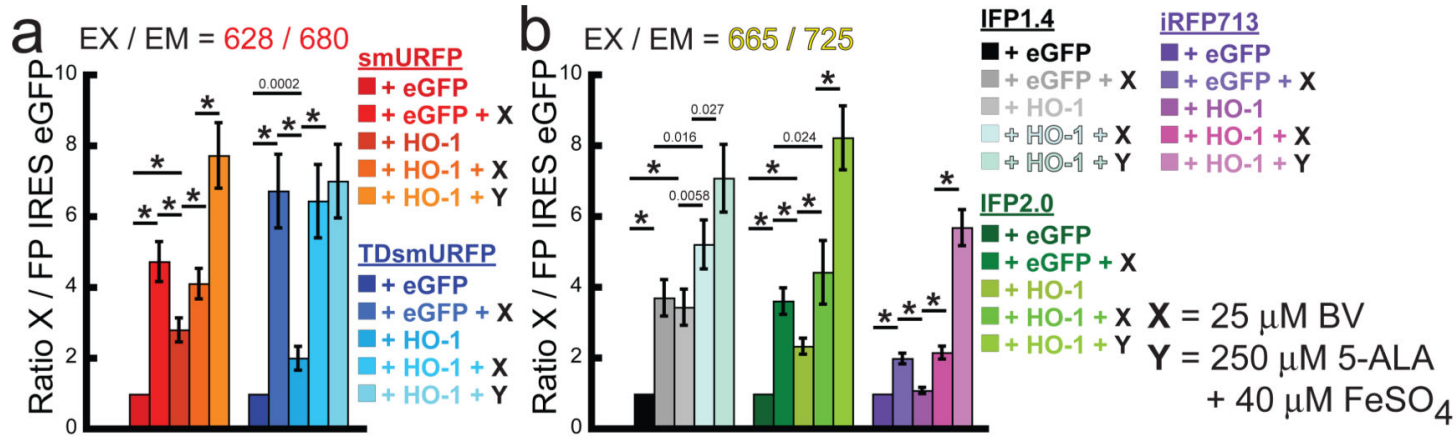

C

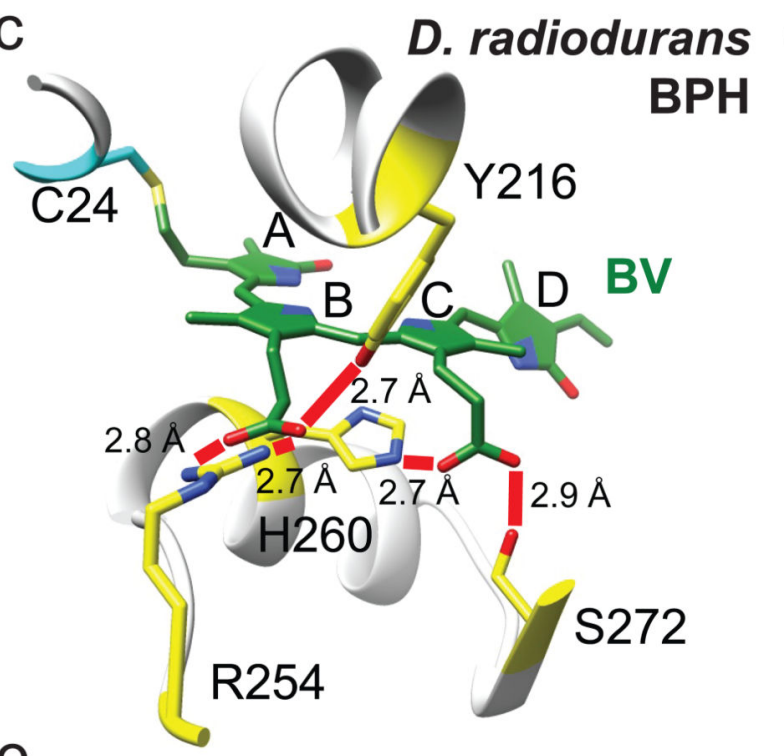

e

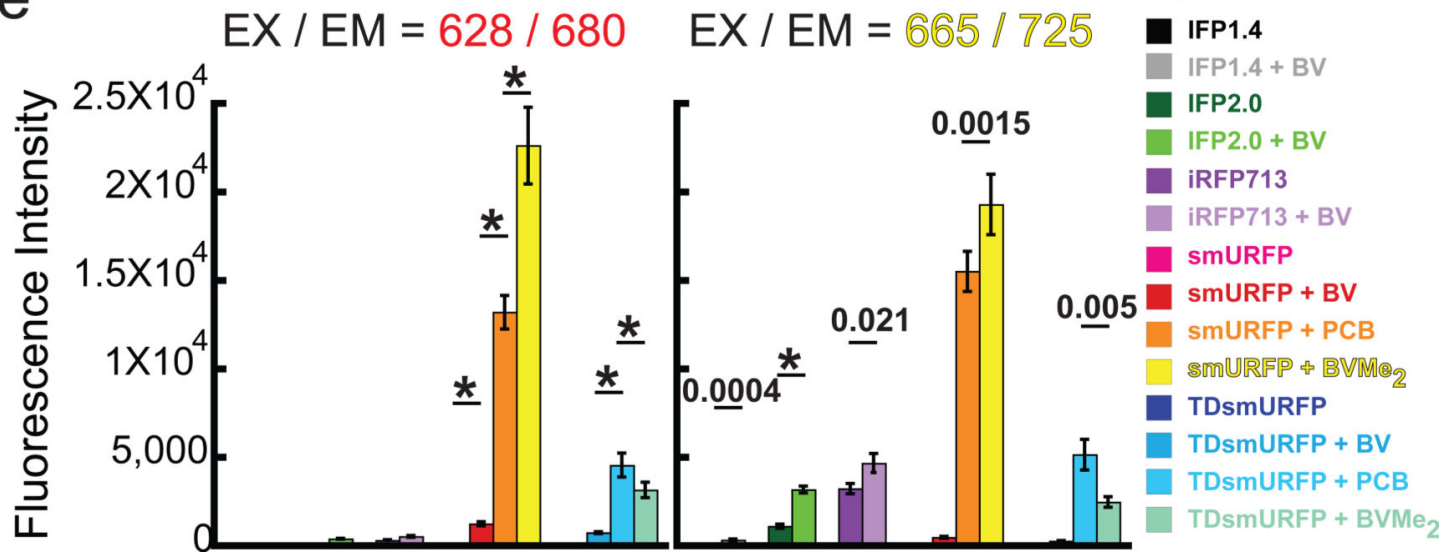

Figure 3.

Increasing chromophore concentration within cells increases fluorescence. HO-1 expression produces BV in situ and increases fluorescence of FPs. (a,b) Quantitation of images in Supplementary Fig. 8. Fluorescence was normalized to FP IRES eGFP without exogenous BV. Expression of HO-1 + 5-ALA $+\mathrm{FeSO}_{4}$ significantly increases fluorescence of all FPs. $\mathrm{BV}$ was added for $3 \mathrm{~h}$ and 5-ALA $+\mathrm{FeSO}_{4}$ for $18 \mathrm{~h}$. Error bars were calculated using error propagation. $P$-values were determined by a one-way ANOVA using the mean fluorescence intensity. (c) Crystal structure of D. radiodurans BPH+BV (parent protein of IFP1.4 and 
IFP2.0). All amino acids $3 \AA$ of BV carboxylic acids are shown in yellow. Carboxylic acid recognition explains why $\mathrm{BVMe}_{2}$ does not bind the BPH FPs. C24 covalent attachment (cyan) and pyrrole rings are designated by letter. Created from 1ZTU.pdb. (d) Homology model of smURFP+BV showing lack of BV carboxylic acid recognition. No amino acid is $\leq 4 \AA$ from the carboxylic acids. C52 covalent attachment (cyan) and pyrrole rings are designated by letter. $\mathrm{BVMe}_{2}$ increases membrane permeability and smURFP/TDsmURFP fluorescence. (e) Quantitation of images in Supplementary Fig. 9. All FPs show significant increased fluorescence with BV. SmURFP+BVMe 2 fluorescence is $>32$-fold increased relative to smURFP and brighter than the BPH FPs even when excited off peak (right). Chromophore incubation time is $3 \mathrm{~h}$. (a,b,e) Only selected significant differences are shown. EX is excitation maximum; EM is emission maximum; error bars are s.e.m.; $n=30$; and $*$ is $P<0.0001$. 

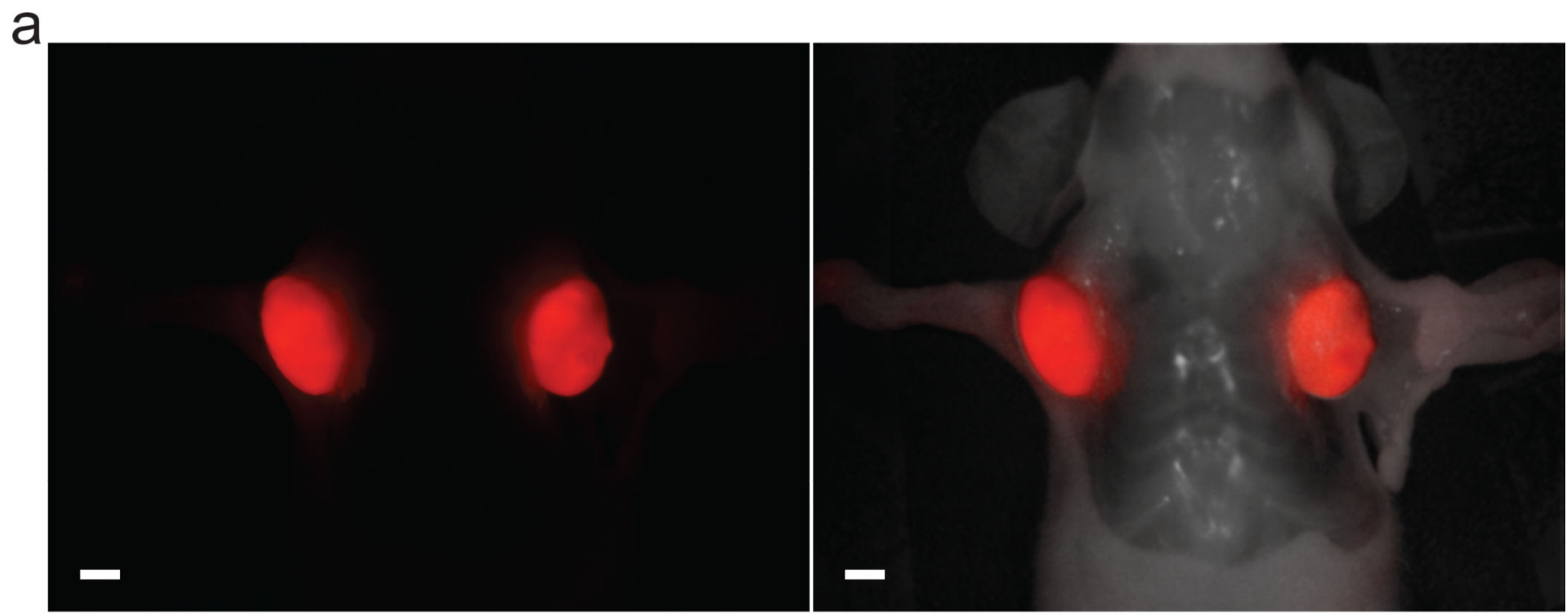

b

C

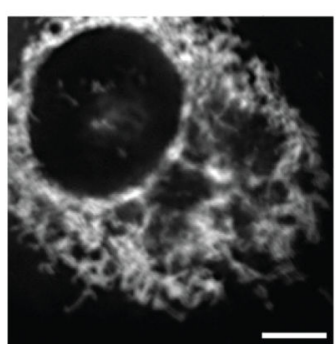

d

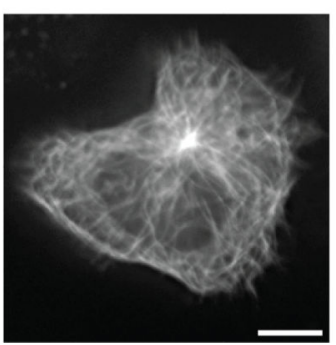

e

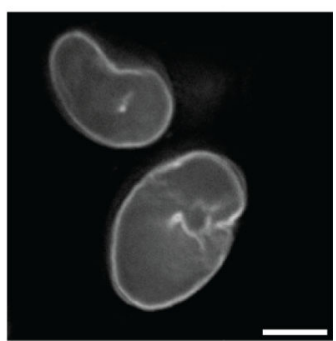

Figure 4.

SmURFP+BV expressed in vivo and smURFP fusions in mammalian cells. (a)

Representative image of smURFP expressed in two HT1080 tumor xenografts without exogenous BV. Fluorescence only (left) and overlay of fluorescence and mouse body (right). Three additional mice are shown in Supplementary Fig. 14. Scale bar $=0.5 \mathrm{~cm}$. (b-d) PC3 cells were transfected with DNA and FP fusions were imaged $48 \mathrm{~h}$ later after incubation with $25 \mu \mathrm{M} \mathrm{BV}$ for $4 \mathrm{~h}$. \#aa is linker length in amino acids and in parentheses: (protein origin, protein name, and cellular location). Fusions at the smURFP N-terminus: (b) ManII-10aa-smURFP+BV (mouse, mannosidase II, and Golgi complex) and (c) PDHA1-10aa-smURFP+BV (human, pyruvate dehydrogenase, and mitochondria). Fusions at the smURFP C-terminus: (d) SmURFP+BV-18aa-aTub (human, a-tubulin, and microtubules) and (e) SmURFP+BV-10aa-LamB1 (human, lamin B1, and nuclear envelope). (b-e) Cell images are representative of $>20$ imaged cells. Similar images were obtained with incubation of $1 \mu \mathrm{M} \mathrm{BVMe}_{2}$. Scale bar $=10 \mu \mathrm{m}$. 


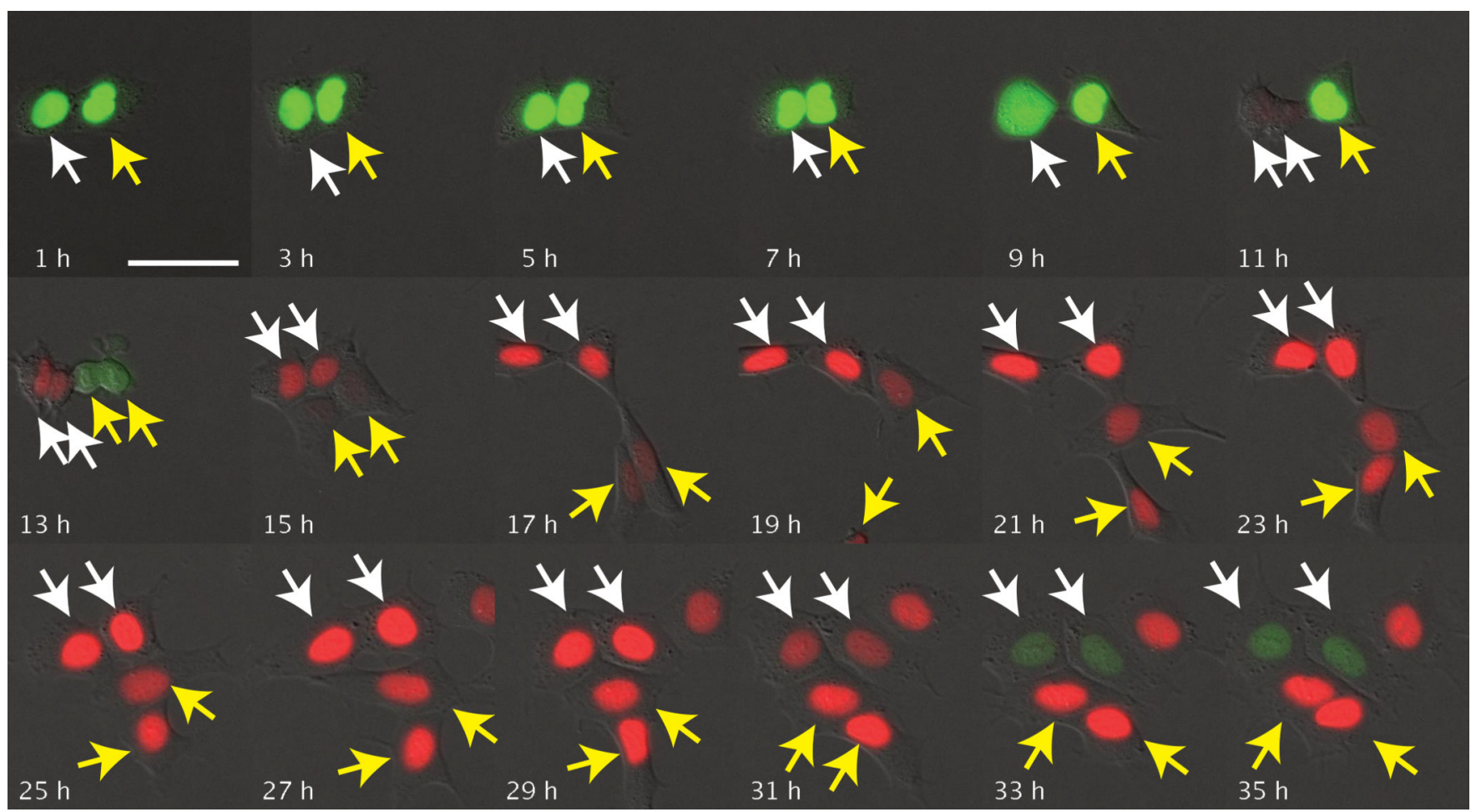

Figure 5.

Time-lapse microscopy of FR/NIR FUCCI expressed in HEK293A cells. IFP2.0hGem(1/110) and smURFP-hCdtI(30/120) fluorescence are shown in green and red, respectively. White and yellow arrows label original cells and their descendants. HEK293A cell division occurs with a doubling time of $\sim 34 \mathrm{~h}$. Green is $\mathrm{EX} / \mathrm{EM}=665(45) / 725(50)$ $\mathrm{nm}$ and red is $\mathrm{EX} / \mathrm{EX}=628(40) / 680(30) \mathrm{nm}$. EX is excitation; $\mathrm{EM}$ is emission; and scale bar $=50 \mu \mathrm{m}$. 


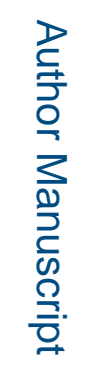

로을

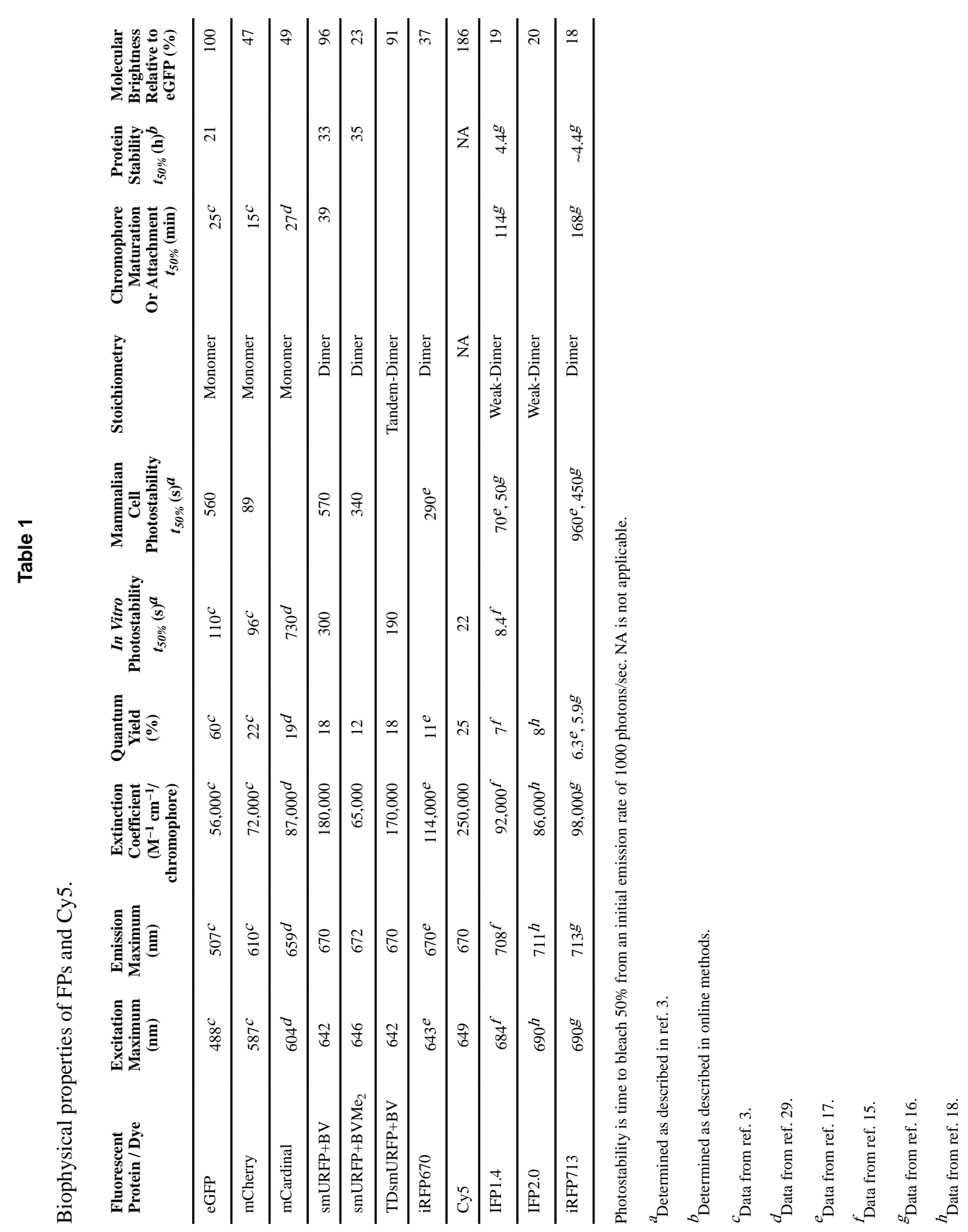

言

동 